\title{
Multiple Regimes and Low-Frequency Oscillations in the Northern Hemisphere's Zonal-Mean Flow
}

\author{
S. Kravtsov and A. W. Robertson* \\ Department of Atmospheric and Oceanic Sciences, and Institute of Geophysics and Planetary Physics, University of California, \\ Los Angeles, Los Angeles, California \\ M. GHIL \\ Department of Atmospheric and Oceanic Sciences, and Institute of Geophysics and Planetary Physics, University of California, \\ Los Angeles, Los Angeles, California, and Département Terre-Atmosphère-Océan and Laboratoire de Météorologie Dynamique \\ du CNRS/IPSL, Ecole Normale Supérieure, Paris, France
}

(Manuscript received 27 October 2004, in final form 8 August 2005)

\begin{abstract}
This paper studies multiple regimes and low-frequency oscillations in the Northern Hemisphere zonalmean zonal flow in winter, using $55 \mathrm{yr}$ of daily observational data. The probability density function estimated in the phase space spanned by the two leading empirical orthogonal functions exhibits two distinct, statistically significant maxima. The two regimes associated with these maxima describe persistent zonalflow states that are characterized by meridional displacements of the midlatitude jet, poleward and equatorward of its time-mean position. The geopotential height anomalies of either regime have a pronounced zonally symmetric component, but largest-amplitude anomalies are located over the Atlantic and Pacific Oceans. High-frequency synoptic transients participate in the maintenance of and transitions between these regimes.

Significant oscillatory components with periods of 147 and 72 days are identified by spectral analysis of the zonal-flow time series. These oscillations are described by singular spectrum analysis and the multitaper method. The 147-day oscillation involves zonal-flow anomalies that propagate poleward, while the 72-day oscillation only manifests northward propagation in the Atlantic sector. Both modes mainly describe changes in the midlatitude jet position and intensity. In the horizontal plane though, the two modes exhibit synchronous centers of action located over the Atlantic and Pacific Oceans. The two persistent flow regimes are associated with slow phases of either oscillation.
\end{abstract}

\section{Introduction}

In this paper, we study the zonally symmetric component of extratropical atmospheric variability in the Northern Hemisphere $(\mathrm{NH})$ using observational data. We concentrate on low-frequency variability (LFV), whose time scales are longer than the few days associated with synoptic eddy evolution.

\footnotetext{
* Current affiliation: International Research Institute for Climate Prediction, Palisades, New York.

Corresponding author address: Dr. Sergey Kravtsov, Dept. of Atmospheric and Oceanic Sciences, University of California, Los Angeles, 405 Hilgard Ave., Los Angeles, CA 90095-1565.

E-mail: sergey@atmos.ucla.edu
}

LFV is characterized by a predominantly equivalent barotropic vertical structure (Wallace 1983). The LFV modes that have a pronounced zonally symmetric component are referred to as annular modes (Wallace 2000). The annular mode in the NH is called the Arctic Oscillation (AO; Deser 2000; Thompson and Wallace 2000; Thompson et al. 2000; Wallace 2000; Robertson 2001), while in the Southern Hemisphere ( $\mathrm{SH})$ it is known as zonal-flow vacillation (Hartmann 1995; Hartmann and Lo 1998; Feldstein and Lee 1998; Lorenz and Hartmann 2001; Koo et al. 2003). Both modes stand out as the leading empirical orthogonal function (EOF) of data that are either low-pass filtered (AO) or zonally averaged (zonal-flow vacillation; see Feldstein and Lee 1998).

The annular modes consist of meridional displacements of the zonally averaged zonal jet (Lorenz and 
Hartmann 2001, 2003). They have also been obtained in idealized numerical models (Robinson 1991, 1996, 2000; Yu and Hartmann 1993; Feldstein and Lee 1996; Koo and Ghil 2002; Kravtsov et al. 2005a). The mechanisms that govern this behavior are not fully understood. Kidson and Watterson (1999) and Feldstein (2000) proposed linear explanations for the variability associated with annular modes. In contrast, Koo et al. (2003) recently presented a nonlinear framework for zonal-flow vacillation, based on the paradigm of multiple weather regimes (Reinhold and Pierrehumbert 1982; Legras and Ghil 1985; Marshall and Molteni 1993). The weather regimes in this context are associated with two persistent zonal-jet states; zonal-flow vacillation is the result of irregular transitions between them due to wave-mean flow interactions.

The two states found by Koo et al. (2003) are associated with distinct probability density function (PDF) maxima in a phase space spanned by the two leading EOFs of the zonal-mean zonal flow. In addition, the time series of the dataset's first principal component (PC-1) exhibits statistically significant spectral peaks representing ultra-low-frequency oscillations. The authors have shown that $\mathrm{SH}$ multiple regimes are associated with extreme phases of these oscillations (Ghil and Robertson 2002), a fact that may provide a basis for improved intraseasonal prediction of weather regimes.

Multiple flow regimes in the $\mathrm{NH}$ have been examined by Ghil and Childress (1987, chapter 6), Mo and Ghil (1988), Cheng and Wallace (1993), Kimoto and Ghil (1993a,b), Plaut and Vautard (1994), and Smyth et al. (1999), among others. They are typically characterized by a large degree of zonal asymmetry; some of the theories for the existence of these regimes involve $\mathrm{NH}$ topography (Charney and DeVore 1979; Kalnay-Rivas and Merkine 1981; Pedlosky 1981; Legras and Ghil 1985; Ghil and Robertson 2000). On the other hand, nonlinear properties of the $\mathrm{NH}$ annular mode have not been studied extensively so far.

In this paper, we perform an analysis of $\mathrm{NH}$ zonalmean zonal flow observations that parallels the one of Koo et al. (2003) for the SH. Zonal averaging is used to exclude from consideration or at least minimize flow signatures associated with lower-boundary forcing, such as topography. We examine evidence for the existence of multiple zonal-flow regimes and lowfrequency oscillations, and the connection between the two, if any.

The paper is organized as follows. The datasets and statistical methodology are described in section 2, while multiple flow regimes, low-frequency oscillations, and the relationship between them are studied in sections 3 , 4 , and 5, respectively. A summary and discussion of the results follow in section 6. Appendix A sets up the stochastic model that serves as the null hypothesis we use for midlatitude LFV, while appendix B studies in greater detail the degree of zonal symmetry of our flow regimes.

\section{Data and methods}

\section{a. Datasets}

In this study, we used averaged daily data for wind $(u, v)$ and geopotential height $Z$ from the National Centers for Environmental Prediction-National Center for Atmospheric Research (NCEP-NCAR) Reanalysis (Kalnay et al. 1996). We analyzed the data subset for $\mathrm{NH}$ winter (1 December-31 March), from December 1948 to March 2003 , on a $2.5^{\circ} \times 2.5^{\circ}$ latitude-longitude grid, using 12 pressure levels $(1000,925,850,700,600$, $500,400,300,250,200,150$, and $100 \mathrm{hPa}$ ) for wind data and three levels $(700,500$, and $250 \mathrm{hPa})$ for geopotential height data. The daily time series thus contained $55 \times 121=6655$ days.

The wind components were decomposed as

$$
u=[u]+u^{*}, \quad v \equiv v^{*},
$$

where square brackets denote the zonal average and a star the deviation from it. Following Lorenz and Hartmann (2003), we divided "eddy" time series $u^{*}$ and $v^{*}$ into three parts, namely the high-frequency, quasistationary, and residual parts:

$$
u^{*}=u_{\mathrm{H}}^{*}+u_{\mathrm{S}}^{*}+u_{\mathrm{R}}^{*}, \quad v^{*}=v_{\mathrm{H}}^{*}+v_{\mathrm{S}}^{*}+v_{\mathrm{R}}^{*} .
$$

The high-frequency and quasi-stationary eddies were found using a 10-day cutoff high-pass and a 40-day cutoff low-pass filters (Otnes and Enochson 1978), respectively. Both filters had 45 weights. The residual eddies were defined as the remainder after the high-frequency and quasi-stationary eddies are removed. The eddy momentum flux $F \equiv\left[u^{*} v^{*}\right]$ was also decomposed as

$$
F=F_{\mathrm{H}}+F_{\mathrm{S}}+F_{\mathrm{R}},
$$

with $F_{H}=\left[u_{\mathrm{H}}^{*} v_{\mathrm{H}}^{*}\right], F_{\mathrm{S}}=\left[u_{\mathrm{S}}^{*} v_{\mathrm{S}}^{*}\right]$, and $F_{\mathrm{R}} \equiv F-F_{\mathrm{H}}-F_{\mathrm{S}}$.

First, the seasonal cycle was removed from the data. This cycle was estimated by applying a 10-day running mean to daily $[u], F_{\mathrm{H}}, F_{\mathrm{S}}, F_{R}$, and $Z$ data and averaging over all years. Next, the El Niño-Southern Oscillation (ENSO) signal was linearly removed from all fields (see Lorenz and Hartmann 2003). This signal was defined by regressing each field onto centered and normalized time series of the Niño-3 index, that is the SST anomaly averaged over the box $5^{\circ} \mathrm{S}-5^{\circ} \mathrm{N}, 150^{\circ}-90^{\circ} \mathrm{W}$. The time series of the daily Niño-3 index was obtained by applying a cubic spline to its monthly time series. We then 

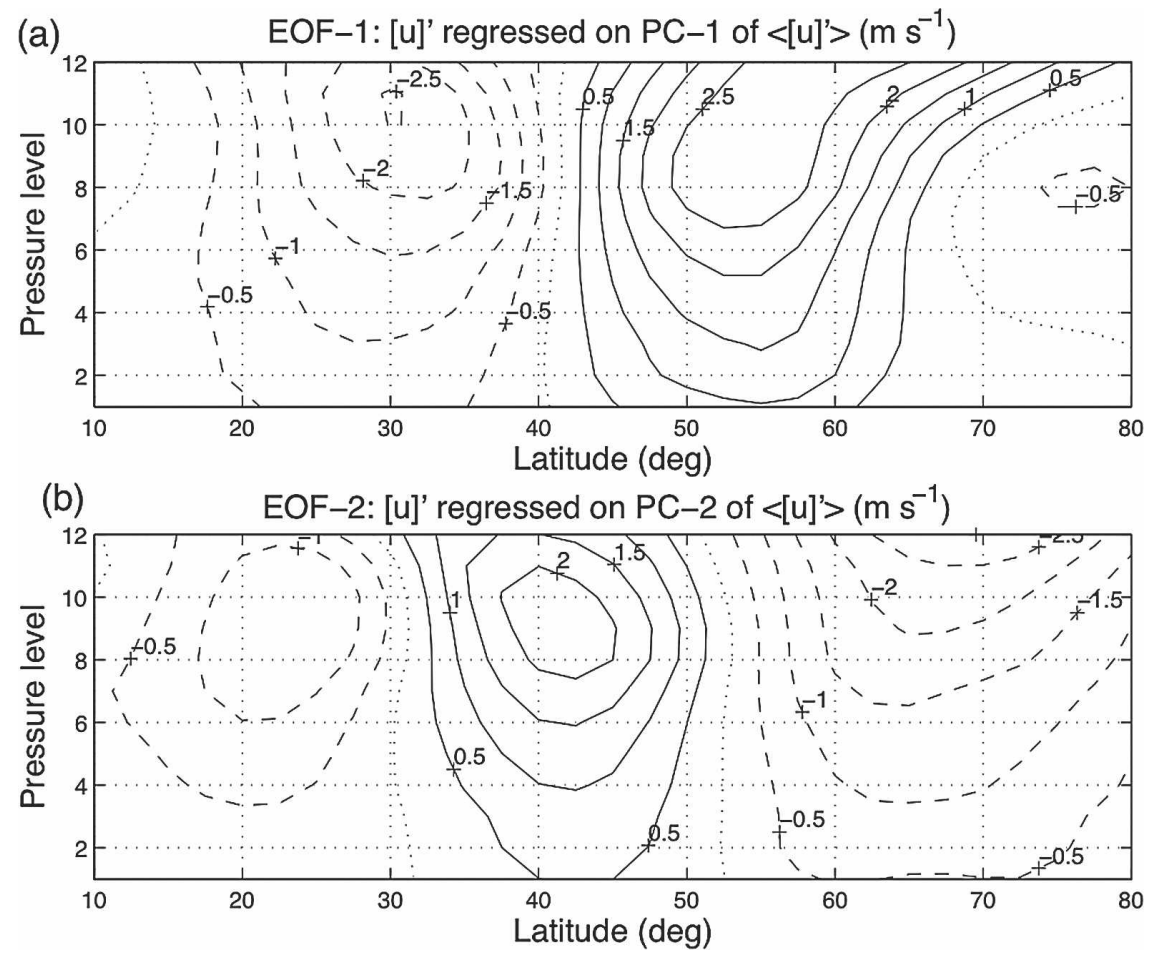

FIG. 1. Leading EOFs of monthly mean zonal wind after linearly removing ENSO variability: (a) zonal-mean zonal wind anomalies $\left(\mathrm{m} \mathrm{s}^{-1}\right)$ regressed on PC-1 of the vertical- and zonal-mean zonal wind using monthly data; (b) same as in (a), but for PC-2.

analyzed the datasets of daily anomalies $[u]^{\prime}, F_{\mathrm{H}}^{\prime}, F_{\mathrm{S}}^{\prime}$, $F_{\mathrm{R}}^{\prime}$, and $Z^{\prime}$ so obtained.

\section{b. EOF analysis}

We performed an EOF analysis of the zonal-wind anomalies $[u]^{\prime}$, obtained as described above. For this analysis, the data fields were weighted by the square root of the cosine of latitude and by the square root of the pressure interval representing each level.

Following Lorenz and Hartmann (2003), EOFs of monthly and vertically averaged anomalies $\left\langle[u]^{\prime}\right\rangle$ were first computed. Monthly anomalies were computed by taking 30-day nonoverlapping boxcar averages of the daily data. Two leading EOFs account for $45 \%$ and $27 \%$ of the dataset's total month-to-month variance, and are well separated from each other, as well as from other EOFs, according to the heuristic test of North et al. (1982). Ten leading EOFs account for a major portion (about $99 \%$ ) of the month-to-month variance.

The two leading EOFs are not sensitive to the particular choice of ENSO index or the method of removing the ENSO signal: Lorenz and Hartmann (2003), who did use a different, multivariate ENSO index, obtained zonal-wind EOFs that are virtually identical to ours. After linearly removing the ENSO signal in both
Lorenz and Hartmann (2003) and in our paper, the centers of action of the leading zonal-wind EOF move poleward by about $2.5^{\circ}$, while the correlation of the leading principal component with the northern annular mode index (Thompson and Wallace 2000) increases from about 0.8 to 0.9 .

Figure 1 shows monthly zonal-wind anomalies regressed on the normalized PC-1 (EOF-1; Fig. 1a) and PC-2 (EOF-2; Fig. 1b) of monthly $\left\langle[u]^{\prime}\right\rangle$. The spatial patterns of these anomalies are very similar to those computed by Lorenz and Hartmann (2003, their Fig. 3) for a shorter dataset (1975-2000). Both modes have an approximately equivalent barotropic vertical structure. The first mode exhibits dipolar variability with opposite anomaly centers located at $30^{\circ}$ and $55^{\circ} \mathrm{N}$. The node is located near $42^{\circ} \mathrm{N}$, which corresponds to the latitude of the maximum low-level winds (not shown). Low-level westerlies are a signature of a midlatitude jet, which is maintained by the eddies; see Lorenz and Hartmann (2003), their Fig. 2. EOF-1 is thus associated primarily with changes in the midlatitude jet position. EOF-2, on the other hand, describes changes in the jet's intensity.

Daily time series of the ten leading EOFs were computed by projecting daily $\left\langle[u]^{\prime}\right\rangle$ anomalies onto the corresponding monthly mean EOFs. The normalized daily 
PCs so obtained were then used for PDF estimation. If full 2D fields are used in the procedure above (not shown), the two leading PCs are virtually identical to those obtained using vertically averaged fields: the correlation between the PC-1s and PC-2s computed by the two methods is 0.98 and 0.96 , respectively.

\section{c. PDF estimation}

To estimate the PDF of zonal-wind data, we employed a multivariate kernel density estimator (Silverman 1986) with an Epanechnikov kernel, as used by Kimoto and Ghil (1983a) and Koo et al. (2003). In this method, the smoothness of the estimated PDF is controlled by the smoothing parameter $h$. Small values of $h$ generally result in spurious PDF maxima, while too large values of $h$ introduce a large bias into the final density estimates. We therefore used trial and error to choose, in each case, an optimal smoothing parameter $h_{o}$ in such a way that the main features of the final density estimate were robust across a moderate range of variations in $h, h_{o}-h^{\prime} \leq h \leq h_{o}+h^{\prime}$, with typical values of $h_{o} \approx 0.5$ and $h^{\prime} \approx 0.1$. The PDF was estimated in a two-dimensional (2D) phase space, spanned by the two leading EOFs of the monthly mean zonal-mean flow, using a Euclidean root-mean-square metric. We also estimated an angular PDF, in which sample maps in physical space are projected onto the unit circle in the same 2D phase space. Such an angular, correlationbased metric emphasizes changes in the zonal-jet profile, rather than changes in the jet strength (Mo and Ghil 1988; Kimoto and Ghil 1993a).

The PDF of the time series generated by a linear Markovian stochastic process forced by Gaussian noise has a normal distribution. Deviations from Gaussianity in the estimated density, for example multiple PDF maxima, thus indicate that nonlinear processes are at work in generating this time series; such processes may include multiplicative noise, as well as purely deterministic nonlinearities. We assessed the statistical significance of such deviations by fitting a multivariate linear stochastic model to the observed time series in the phase space of the ten leading EOFs of the zonal-mean flow (see appendix A). This model (Kravtsov et al. 2005b) is a multilevel generalization of the first-order autoregressive [AR(1)] model used by Koo et al. (2003) for the same purpose. One hundred realizations of the time series generated by this stochastic model, each having the same length as the PCs of the dataset were generated and used to compute one hundred random PDFs with the same $h$ as for the data. The percentage of random PDFs that fall short of the dataset's PDF values gives an estimate of the statistical significance with respect to our null hypothesis.

\section{d. Spectral analysis methods}

To examine the temporal behavior of the $\mathrm{NH}$ zonal flow, we adopted two complementary methods of spectral analysis (Ghil et al. 2002): the multitaper method (MTM; Thomson 1982, 1990; Mann and Lees 1996) and the multichannel version of singular-spectrum analysis (SSA; Broomhead and King 1986; Fraedrich 1986; Vautard and Ghil 1989; Vautard et al. 1992). MTM uses a small set of optimal windows, or tapers, that objectively minimize power leakage and reduce uncertainties in the estimation of spectral density. Statistical significance against a red-noise null hypothesis is assessed by fitting an AR(1) process to the time series being tested (Mann and Lees 1996).

Multichannel SSA (M-SSA) generalizes singlechannel SSA to extract oscillatory modes of variability from a multivariate time series (Keppenne and Ghil 1993; Plaut and Vautard 1994). M-SSA finds eigenvalues and eigenvectors of the grand covariance matrix $\mathbf{C}$ computed for an augmented vector time series; the latter consists of the original vector time series and $M$ lagged copies thereof. The window size $M$ determines the range of periodicities that may be detected. The eigenvalues of $\mathbf{C}$ are called the space-time EOFs or ST-EOFs; the associated space-time PCs (ST-PCs) are single-channel time series that are computed by projecting augmented vector time series onto the STEOFs. M-SSA identifies an oscillation in a multivariate time series when two consecutive eigenvalues of $\mathbf{C}$ (ordered by size) are nearly equal, the corresponding STEOFs are periodic, with the same period and in quadrature, while the associated ST-PCs are in quadrature as well. Following Allen and Robertson (1996) we apply, in addition to the criteria above, a Monte Carlo test to ascertain the statistical significance of the oscillation detected by M-SSA.

When the methods described above identified multiple flow regimes or low-frequency oscillations, we performed a composite analysis keyed to a given regime or a given phase of a low-frequency oscillation to describe the anomalies in the physical space associated with them (Ghil and Mo 1991a,b; Plaut and Vautard 1994). The statistical significance of each composite anomaly field was estimated using a nonparametric Monte Carlo method (Dole and Gordon 1983; Vautard et al. 1990; Plaut and Vautard 1994). First, we gathered into time segments the consecutive days belonging to a given oscillation phase or a given regime, including a null regime defined as all data points that do not belong to any of the regimes identified. These segments were randomly shuffled 100 times, thus providing 100 independent realizations with the same length. Each quan- 


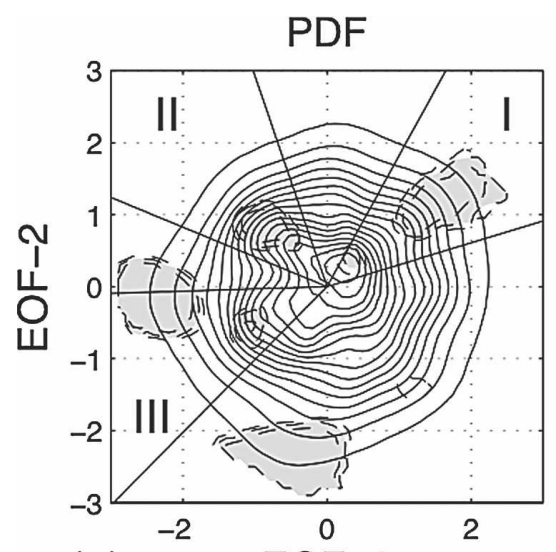

(a)

EOF-1

Angular PDF

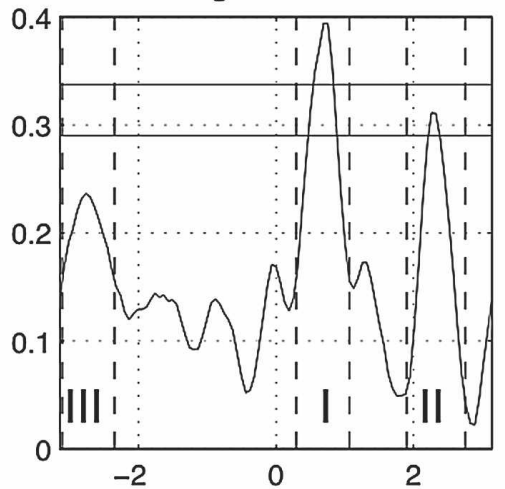

(c)
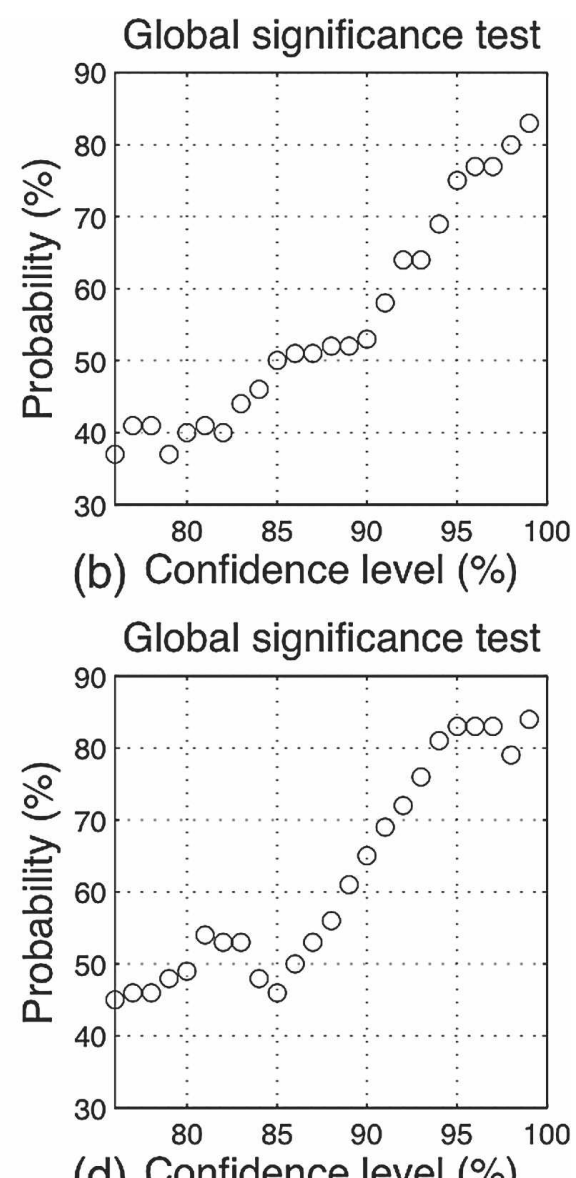

FIG. 2. Probability density estimation for the entire dataset: (a) Two-dimensional PDF (solid contours) in a plane spanned by EOF-1 and EOF-2, with a smoothing parameter of $h=0.55$. Dashed contours show the confidence levels, that is the number, out of 100, of random PDFs (see text for details) that fall short of the estimated PDF values for the data; contours are 90, 95, and 99, the regions with values larger than 95 are shaded. (c) Light straight lines bound the regime sectors that are defined. (b) Global significance test (see text for details). (c) Angular PDF in the same plane as in (a), with smoothing parameter $h=0.4 \mathrm{rad}$; horizontal solid lines show $95 \%$ and $99 \%$ confidence levels, while vertical dashed lines define the boundaries of the sectorial regimes. (d) Same as in (b), but for the angular PDF estimate.

tity that was estimated for a given composite, whether keyed to a given regime or to an oscillation's given phase category, was also computed using the 100 shuffled sets of category numbers. The $95 \%$ confidence interval, for example, is then bounded by the 5 th and 95th percentiles of the random values so computed, sorted in ascending order.

\section{Probability density estimation}

\section{a. PDF estimates for the entire dataset}

We estimated the 2D PDF of the daily zonal-mean flow anomalies as described in section $2 \mathrm{c}$. The results obtained with a smoothing parameter $h=0.55$ are shown in Fig. 2a. The PDF has an overall circular shape, but its global maximum, which is statistically significant, is located slightly off-center. Three statistically significant ridges are identified in the first, second, and third quadrants.

In addition to a local test of statistical significance (see section 2c), we also perform, following Koo et al. (2003), a global significance test because of Hsu and Zwiers (2001); the latter test determines whether significant local departures from a Gaussian PDF contribute a globally significant amount of probability. To do so, one integrates an estimated PDF over the areas identified as significant in the local test, and ranks this 


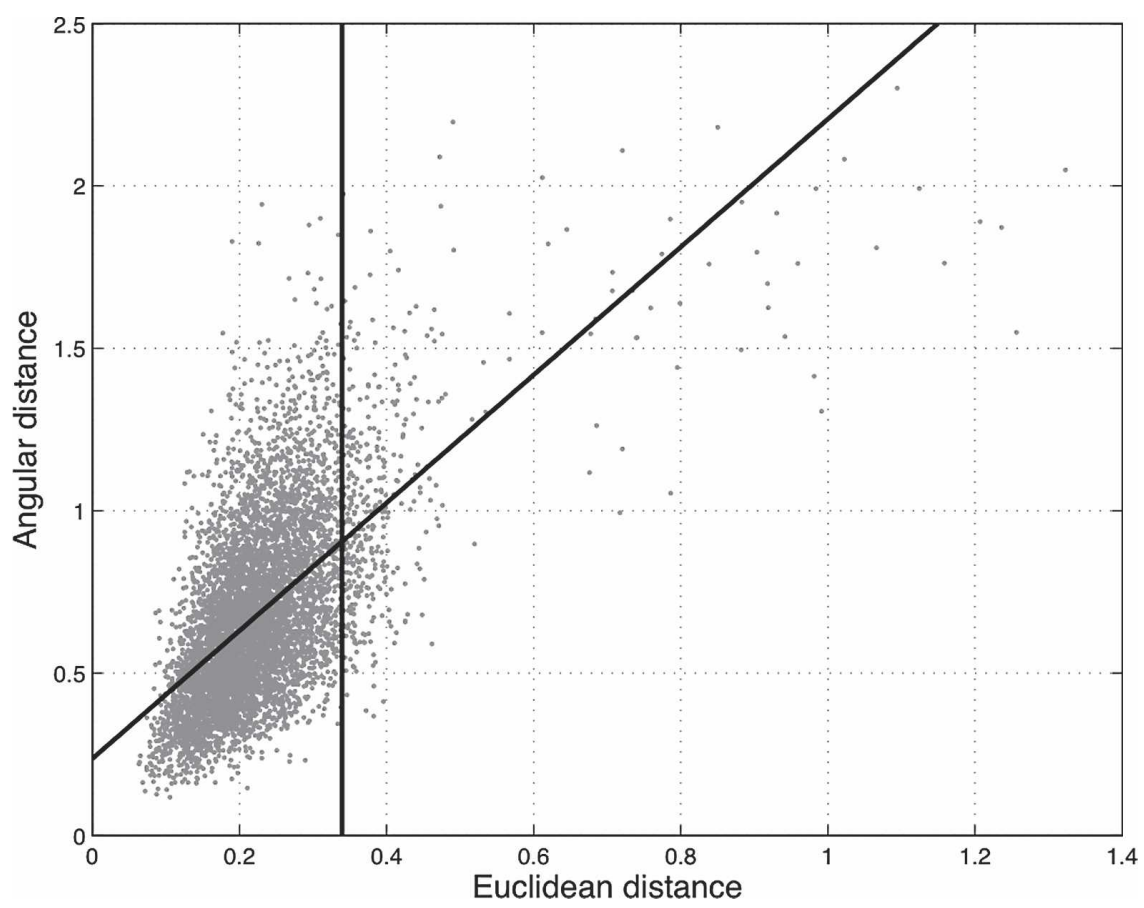

FIG. 3. Data scatter in terms of two distance measures: the sloping solid line indicates a least squares linear fit; the vertical line denotes 1.5 times the mean value of the Euclidean distance. The QS subset consists of data that lie to the left of the vertical line and below the sloping one.

statistic, called an IPDF, against the probabilities obtained by integrating the synthetic PDFs over their respective areas of local significance. The results of this test are shown in Fig. 2b, which shows the number, out of 100 , of synthetic PDFs that have an integrated probability lower than that of the data, as a function of local significance level. We find that the areas that are locally significant at the $95 \%$ level and higher are also likely to be globally significant, with a high probability of about $80 \%$.

Significant regions in Fig. 2a, aside from the global maximum located close to the center, appear as three elongated ridges. We therefore computed an angular PDF, which is shown in Fig. 2c. The angle is measured from the positive EOF-1 axis, and we used the smoothing parameter of $h_{o}=0.4 \mathrm{rad}$. Three PDF maxima are identified, two of which are significant globally, as well as locally (see Figs. 2c,d).

The sectors shown in Figs. 2a,c define the boundaries of the sectorial regimes. We collected the data points that belong to each regime, and computed corresponding distributions of residence times (not shown). The mean residence time for each of the regimes I, II, or III in the synthetic dataset is of about 3.5 days, while the $95 \%$ confidence level corresponds to a residence time of about 4.5 days. The residence times for regimes I, II, and III in our $\mathrm{NH}$ dataset are of about 4.7 days. We thus find that all three regimes are associated with anomalously persistent flow patterns: their residence times are significantly longer than those of their synthetic random counterparts, at the $95 \%$ confidence level. To examine if this increased persistence is associated with particular geographical flow patterns, we consider next quasi-stationary (QS) subsets of the full dataset, as suggested by Mo and Ghil (1988).

\section{b. PDF estimates for quasi-stationary data}

To obtain QS subsets of the data, we used two different methods. The first one, due to Koo et al. (2003), computes the Euclidean, as well as the angular distance between two samples one day apart, in the 10D phase space defined in section $2 \mathrm{~b}$. The Euclidean distance $D$ and the angular distance $\phi$ between two vectors $\mathbf{x}$ and $\mathbf{y}$ are given, respectively, by

$$
D \equiv|\mathbf{x}-\mathbf{y}|, \quad \cos \phi \equiv \frac{\mathbf{x} \cdot \mathbf{y}}{|\mathbf{x}||\mathbf{y}|} .
$$

Here the dot denotes the scalar product $\mathbf{x} \cdot \mathbf{y} \equiv \Sigma_{i} x_{i} y_{i}$, while $|\mathbf{x}|^{2} \equiv \mathbf{x} \cdot \mathbf{x}$. The relationship between these two measures, for all pairs of successive maps, is shown in Fig. 3 as a scatterplot. The QS subset was defined by collecting the points that fall into the lower left corner, below the straight regression line and to the left of the 
vertical at 1.5 times the mean Euclidean distance. The resulting QS subset consists of 3493 maps, which constitutes roughly half of the original dataset.

The other method (Legras and Ghil 1985; Vautard et al. 1988) concentrates on persistent sequences of anomalies. For each record $t$, and for a given characteristic time $T$, a persistence score $s(t, T)$ was defined as

$$
s(t, T)=\max \left[A\left(t^{\prime}\right): t \leq t^{\prime} \leq t+T\right],
$$

where $A\left(t^{\prime}\right)$ is the Euclidean distance, in the same 10D phase space, between two maps sampled at times $t$ and $t^{\prime}$, respectively. Thus, for large enough $T$, a low score $s(t, T)$ characterizes a sequence during which the flow pattern does not change dramatically. We used the value of $T=10$ days; the results do not change qualitatively if larger or smaller values of $T$, from 8 to 12 days, are used. A persistent dataset was defined as the one that consists of sequences whose score is lower than the mean score computed over all sequences; this subset has 3632 data points.

The QS and persistent subsets defined above have about the same number of points, roughly 3500 versus 3600; still, they emphasize somewhat different dynamical regimes. The former collects pairs of consecutive maps that are both well correlated and close to each other in terms of Euclidean distance; the resulting subset thus concentrates on recurrent, but not necessarily persistent anomalies that, moreover, may have a relatively large amplitude (Koo et al. 2003). In contrast, the persistent subset is biased toward small-amplitude anomalies, since it employs a Euclidean-distance measure only, while it considers only the patterns characterized by a relatively long lifetime of about 10 days.

We compute next PDFs of the QS and persistent data subsets. To estimate the statistical significance of the resulting PDF, the surrogate random time series defined in section $2 \mathrm{c}$ were subsampled in the same way as the QS and persistent data subsets, and random PDFs were computed with the same smoothing parameter as for the observed data. The PDF for the QS subset estimated with the smoothing parameter of $h_{o}=0.6$ is shown in Fig. 4a. It has a bimodal distribution with the main maximum close to the global maximum in the PDF of the full dataset (Fig. 2a), and a secondary maximum in the third quadrant that corresponds to a statistically significant ridge in Fig. 2a. The two maxima are statistically significant, both locally and globally (Fig. $4 b)$.

The PDF of the persistent data subset (not shown), on the other hand, is qualitatively very similar to the full dataset's PDF (Fig. 2a), but with the statistically significant ridges being more pronounced in the former. Following Vautard et al. (1988), we computed the per- sistence probability as the ratio between the subsampled and full data PDFs, each weighted by the total number of records in the corresponding dataset. This distribution is shown in Fig. $4 \mathrm{c}$ and is, once again, significantly bimodal; moreover, the two persistence maxima are located close to the phase-space density maxima in Fig. 4a. The persistence maxima associated with regimes $\mathrm{A}$ and $\mathrm{B}$ are characterized by a persistence probability of about $70 \%$ and $65 \%$, respectively.

To identify the spatial patterns associated with each PDF maximum, we collected all the points in the full dataset that fall within the two ellipses A and B in Figs. 4a,c. A total of 1362 and 1102 days are assigned to regimes $\mathrm{A}$ and $\mathrm{B}$, respectively. The semiaxes of the two ellipses here were chosen subjectively, given the small amount of observational data, to achieve a compromise between statistical significance and closeness to the PDF maxima for each regime. Kondrashov et al. (2004) have explored the sensitivity of results on the size of the ellipses in a very long simulation (54 000 days) of an intermediate-complexity model (Marshall and Molteni 1993) and found relatively little sensitivity to the definition of the regime regions.

The distribution of residence times for both regimes is shown in Fig. 4d. Both regimes are characterized by anomalies that are significantly more persistent than those computed for the random data surrogates. Regime $\mathrm{B}$ anomalies, however, are more persistent than those of regime A, despite the former being less frequently visited: the average residence time for regime A is 3 days, while it equals to 3.4 days for regime B.

\section{c. Regime composites}

Zonal-flow composites for regimes A and B are plotted in Figs. 5a,b, respectively. Regime A is associated with a poleward and regime $\mathrm{B}$ with an equatorward shift of the zonal midlatitude jet, away from its climatological position; both regimes have a nearly equivalent barotropic vertical structure, as expected.

Composites of the 700-hPa geopotential height anomalies for regimes A and B are shown in Figs. 6a,b. The spatial patterns of the 500- and 250-hPa anomalies (not shown) are very similar to those at $700 \mathrm{hPa}$, in agreement with the equivalent barotropic character of NH LFV. The two regimes have a large degree of zonal symmetry and are thus possibly related to the two opposite phases of the AO (Deser 2000; Thompson and Wallace 2000; Thompson et al. 2000). Largest amplitude anomalies for both regimes are located over the North Atlantic and North Pacific Oceans, with a belt of opposite-sign anomalies over the Arctic Ocean. We demonstrate in appendix B that the set of flow maps for regime $\mathrm{B}$ is indeed dominated by anomalies of the same 

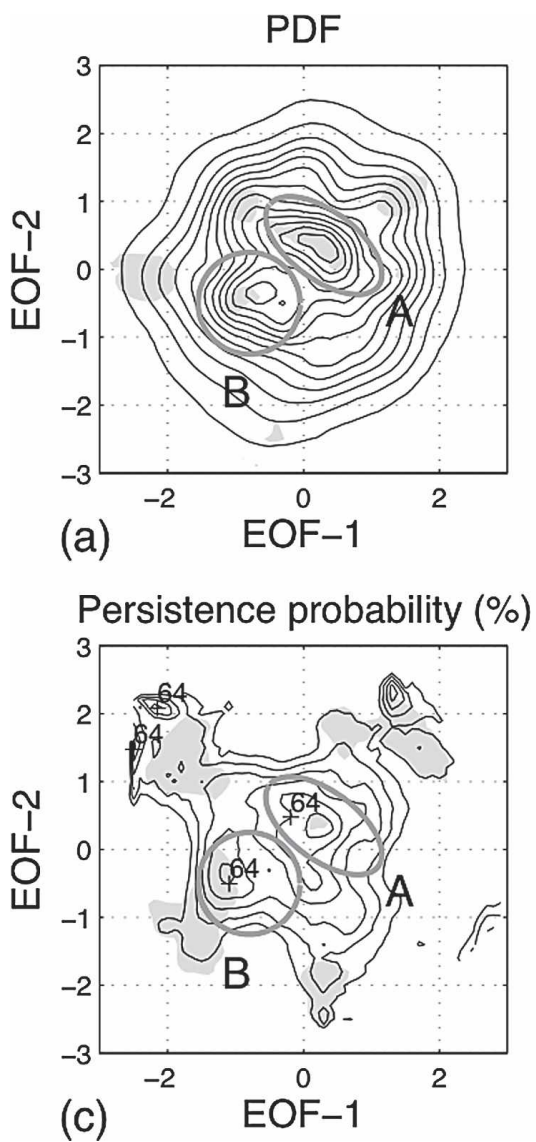
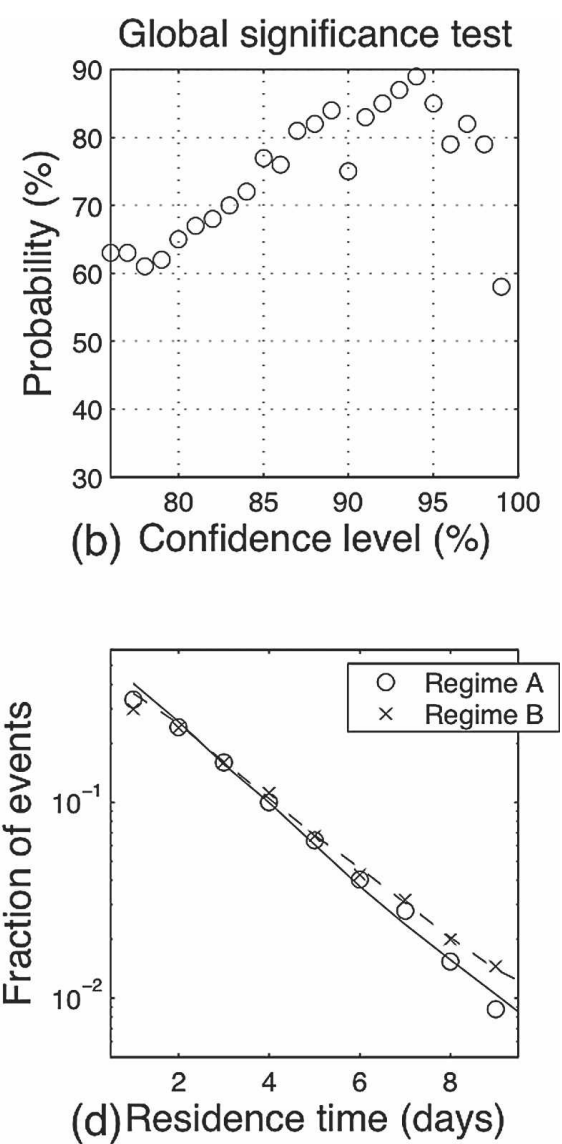

FIG. 4. PDF estimation for data subsets: (a), (b) Same as in Figs. 2a,b, but for the QS data defined in Fig. 3; smoothing parameter is $h=0.6$. (c) Persistence probability (\%), that is 100 times the ratio of the persistent data subset's PDF (see text) to the full dataset's PDFs; both PDFs are estimated with a smoothing parameter of $h=0.55$ and weighted by the total number of data points belonging to each dataset. In (a) and (c), shading indicates areas that are locally statistically significant at the $95 \%$ level, while the two ellipses define the boundaries of distinct flow regimes. (d) Distribution of cumulative residence times in the two regimes (symbols), as well as 95th percentile of the residence-time distribution within a population of 100 random surrogate data samples (lines). Circles and solid line represent regime $\mathrm{A} ; \times$ symbols and dashed line represent regime B.

sign and comparable magnitude over the Atlantic and Pacific sectors, while for regime A the maps can be divided into two main groups, each characterized by large anomalies in one of the sectors and smaller anomalies in the other.

The composites of zonally and vertically averaged zonal-flow anomalies and eddy forcing anomalies (see section 2a) associated with each regime are shown in Figs. 7a,c for regime A and in Figs. 7b,d for regime B. The high-frequency synoptic transients maintain the anomalies in either regime, while lower frequency residual eddies damp them. Our results here, too, are consistent with previous studies (Feldstein and Lee 1996, 1998; Koo 2001; Koo et al. 2003; Lorenz and Hartmann 2003). The QS eddy forcing has a large positive projection on regime anomalies, but tends also to induce poleward drift of these anomalies (see also Feldstein 1998; Lorenz and Hartmann 2003). Closer inspection shows that the QS eddy flux is largely dominated by the term $\left\langle\left[u_{r}^{*} \overline{v^{*}}+\overline{u^{*}} v_{r}^{*}\right]\right\rangle$, where $u_{r}^{*}, v_{r}^{*}$ and $\overline{u^{*}}, \overline{v^{*}}$ are deviations from zonal mean of regime anomalies and climatological wind, respectively.

\section{Spectral analysis}

To focus on intraseasonal time scales, we took 5-day nonoverlapping boxcar averages of the PC-1 and PC-2 time series. We then applied two-channel M-SSA to the two time series so obtained and MTM to the first one alone. In M-SSA, we chose a window of $M=73$ lags to 


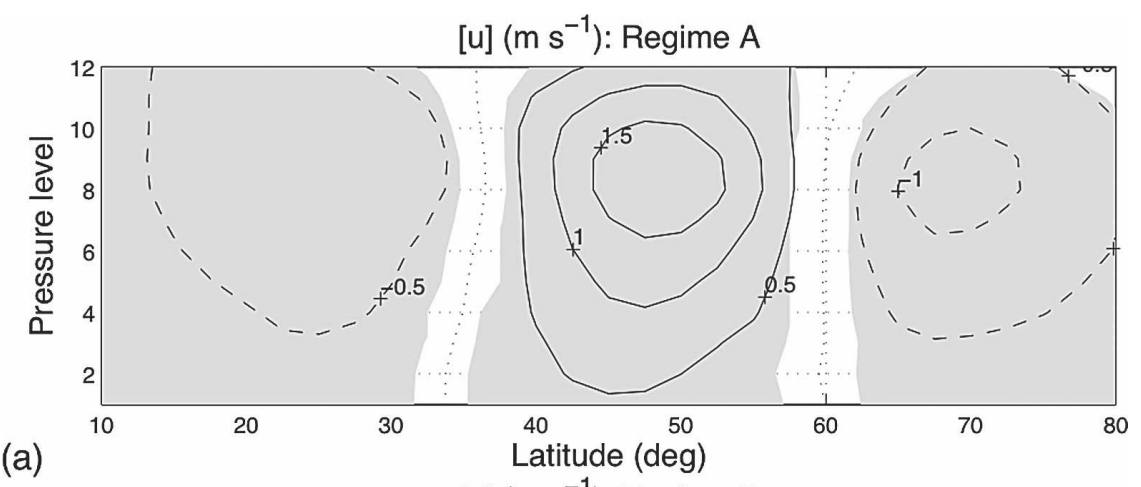

[u] $\left(\mathrm{m} \mathrm{s}^{-1}\right)$ : Regime B

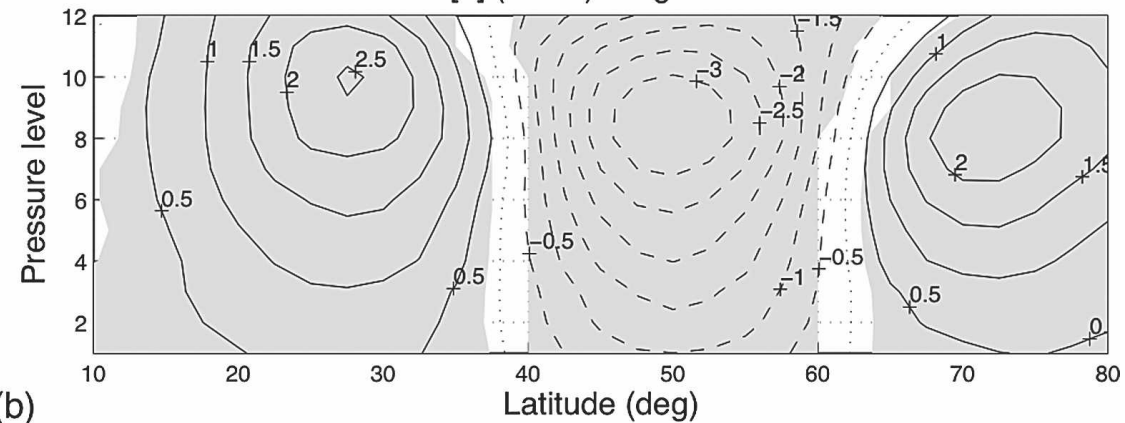

FIG. 5. Composites of zonal-mean flow anomalies $\left(\mathrm{m} \mathrm{s}^{-1}\right)$ for (a) regime $\mathrm{A}$ and (b) regime B days. Solid contours are positive (eastward flow) and dashed ones are negative (westward flow); shading indicates areas with values that are statistically significant at the $95 \%$ level.

target intraseasonal oscillatory modes; this corresponds to a dimensional window width of 365 days and gives an approximate spectral resolution of $1 / M=0.014$ cycle $(5$ days $)^{-1}$. In MTM, we used nine tapers, which yields a half-bandwidth resolution of 0.0075 cycle $(5 \text { days })^{-1}$. These resolutions correspond to frequencies of about $2.7 \times 10^{-3}$ day $^{-1}$ and $1.4 \times 10^{-3}$ day $^{-1}$, respectively.
The resulting spectra are shown in Fig. 8. Two significant oscillations, with periods of 147 and 72 days, are identified by both methods. To confirm that the periodic signals we have obtained are not artifacts of the data preprocessing, we have also performed MTM analysis of the time series obtained by projecting the entire dataset of raw, daily zonal winds for all seasons

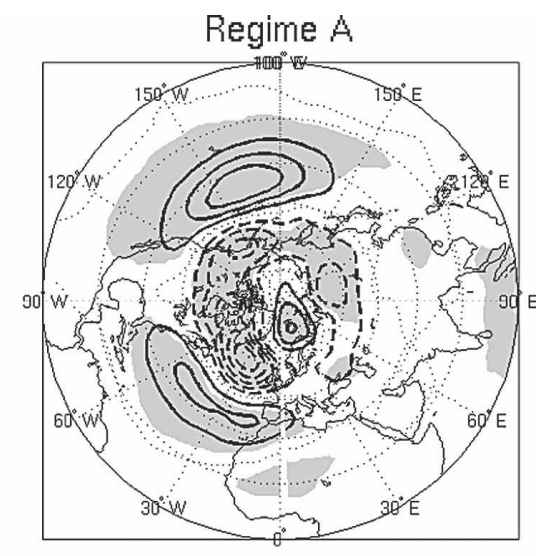

(a)

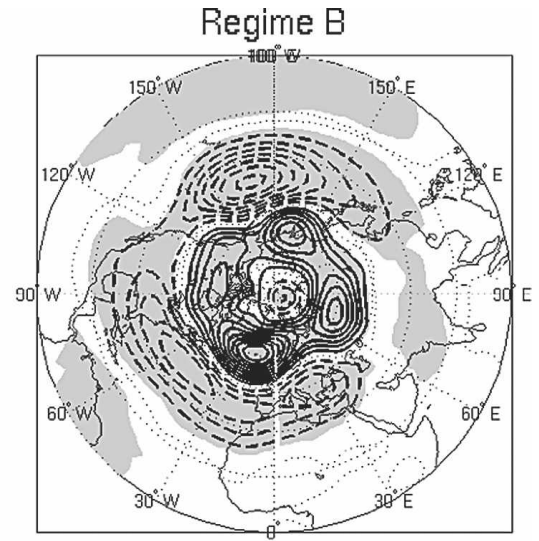

(b)

FIG. 6. Same as in Fig. 5, but for 700-hPa geopotential height anomalies (m). Contour interval $(\mathrm{CI})=5 \mathrm{~m}$; negative contours dashed, zero contour dotted. 


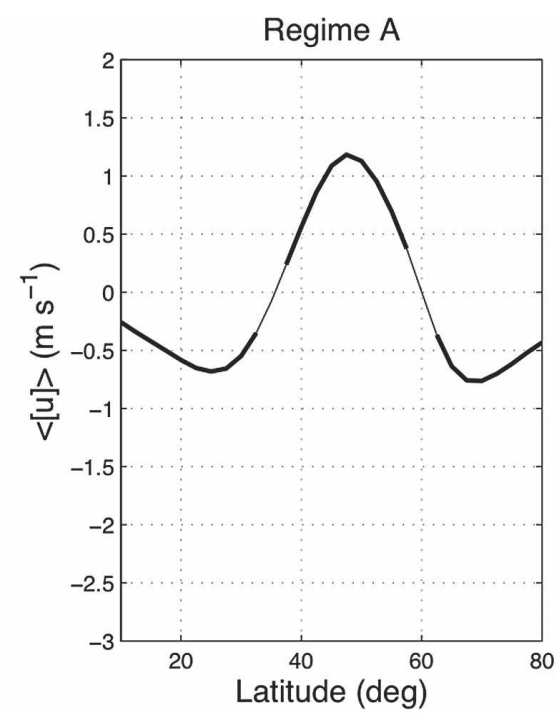

(a)

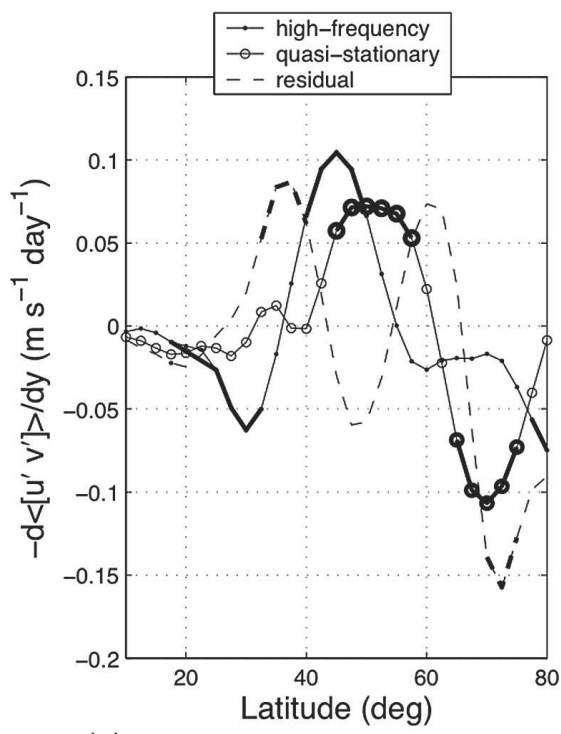

(c)

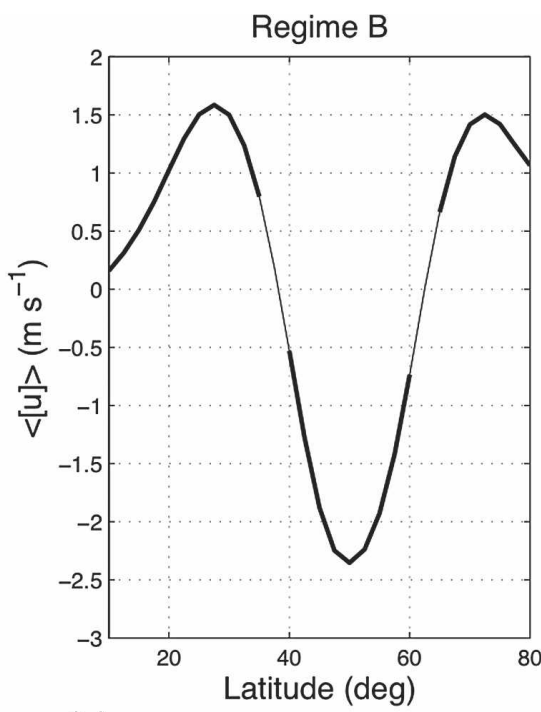

(b)

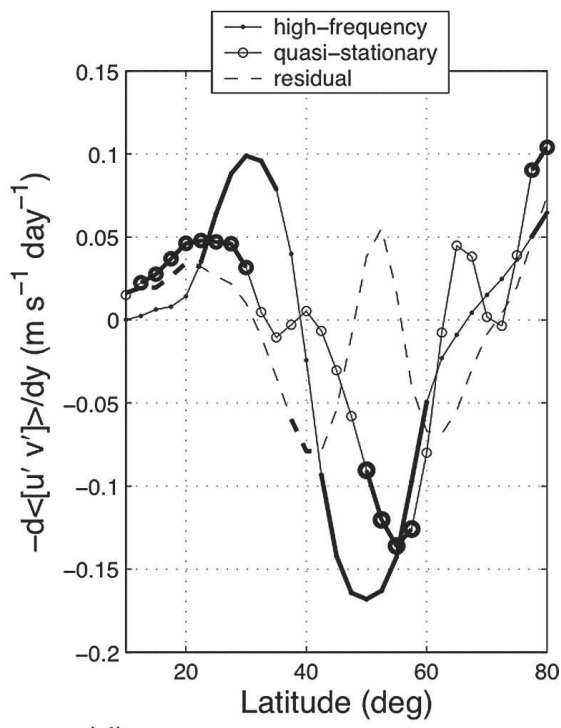

(d)

FIG. 7. Same as in Figs. 5 and 6, but for the (a), (b) vertical- and zonal-mean zonal velocity $\left(\mathrm{m} \mathrm{s}^{-1}\right)$; and (c), (d) minus the divergence of the vertical- and zonal-mean eddy momentum flux components $\left(\mathrm{m} \mathrm{s}^{-1} \mathrm{day}^{-1}\right)$. Lines are defined in the legend, values that are statistically significant at the $95 \%$ level are highlighted.

onto the leading EOF of filtered wintertime anomalies (Fig. 1a). The same peaks with periods of 147 and 72 days were obtained, in addition to those that represent the first two harmonics of the annual cycle (Fig. 9a). These signals were found to be statistically significant at the $99 \%$ level against a red-noise null hypothesis.

The spectral resolution used in Fig. 9a corresponds to about 100 degrees of freedom. We may expect, therefore, at least one spurious spectral peak to exceed the $99 \%$ confidence level and five such peaks to exceed the $95 \%$ level. To overcome the reasonable doubts raised by this consideration, we note that the spectral peaks at 72 and 147 days are confirmed further by using two different types of spectral analysis (SSA and MTM) and applying them to both raw and filtered datasets, that is, with and without the seasonal cycle, respectively.

Figure 9b shows an arbitrarily chosen 1500-day-long segment of the 147-day oscillation's SSA reconstruction, along with the raw data time series; both have been projected onto EOF-1 and the seasonal cycle was removed from the latter. The oscillation persists throughout all seasons. 


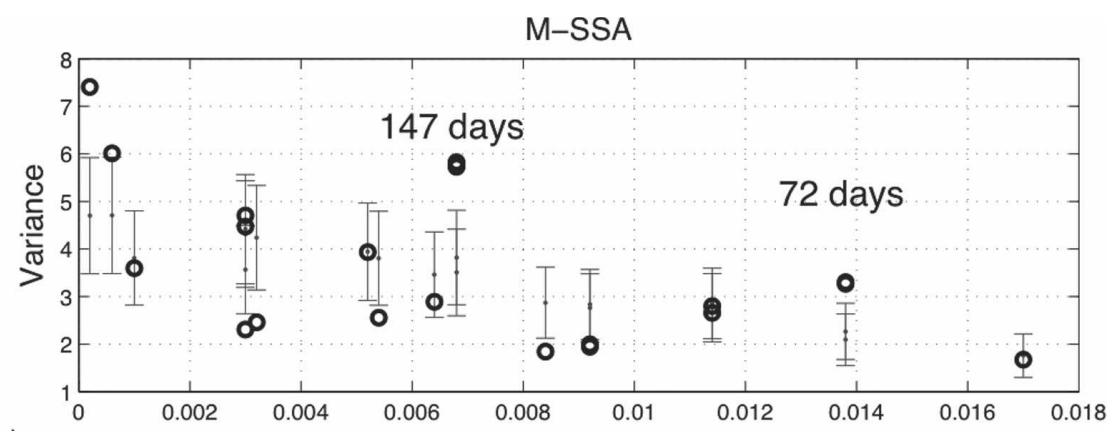

(a)

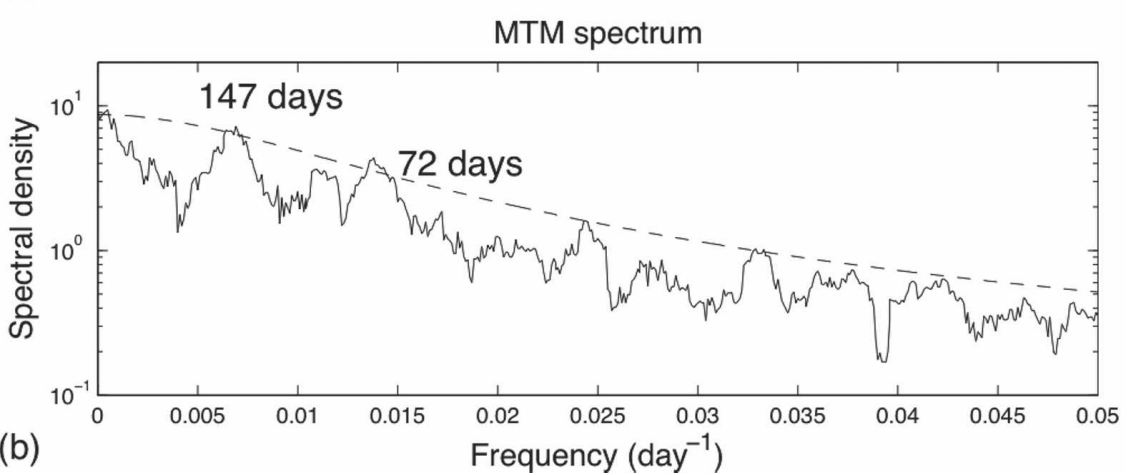

FIG. 8. Power spectra of zonal-mean flow. (a) Singular spectrum obtained by M-SSA of data in the EOF-1-EOF-2 subspace. Circles show M-SSA eigenvalues, plotted against the dominant frequency associated with the corresponding space-time PCs. Two significant pairs with periods of 147 and 72 days are identified; note that the two circles in either pair are almost indistinguishable. The confidence intervals correspond to the 5th and 95th percentiles of 100 Monte Carlo realizations of a red-noise process with the same length, variance and lag-1 autocorrelation as the data time series being tested; see text and section 4.2 of Ghil et al. (2002) for details. (b) Spectrum of PC-1 estimated by the MTM; solid lines are spectral estimates, while dashed line represents a $99 \%$ confidence level with respect to a red-noise null hypothesis. The two most salient oscillatory signals have the same periods as in (a).

We next assigned to each record in the observationbased time series a number, from 1 to 8 , which represents a phase category for a given oscillation. To do so, we define $Y(t)$ to be the channel-one time series of an M-SSA reconstruction (Keppenne and Ghil 1993; Plaut and Vautard 1994) for the oscillation under consideration, and $\dot{Y}(t)$ to be its local tendency. The time series $Y$ for a given oscillation is based on the pair of reconstructed components (RCs) that represents it. The RC pairs are narrow-band versions of the time series, where the filters are derived data-adaptively from the time series itself in order to maximize the variance captured. The two RC pairs account for $28 \%$ and $22 \%$ of the variance of the PC- 1 time series and for $15 \%$ and $10 \%$ of the variance of the PC- 2 time series, for the 147-day and 72-day oscillations, respectively. The eight phase categories were defined by dividing the time series of the angle $0 \leq \phi<2 \pi$ between the instantaneous vector $(Y, \dot{Y})$ on day $t$ and the vector $(0,1)$ into eight equally populated segments.
To check whether the 72-day oscillation is a harmonic of the 147-day oscillation, we counted, over the whole time series, the number $N_{m n}$ of simultaneous occurrences of phase category $m$ of one oscillation and phase category $n$ of the other $(m=1,8 ; n=1,8)$, following Plaut and Vautard (1994), Dettinger and Ghil (1998), and Moron et al. (1998). If the two oscillations are phase locked, each phase of the longer period oscillation must preferentially contain two consecutive phases of the shorter period one. In contrast, we find that $N_{m n}$ is not so structured, while only a few entries in $N_{m n}$ are statistically significant at the $95 \%$ level according to the nonparametric Monte Carlo test described in section $2 \mathrm{~d}$. We conclude, therefore, that the two oscillations identified by our spectral analysis are not phase locked, nor is either one of them phase locked to the seasonal cycle.

The composite cycle of the 147-day oscillation in zonal flow is shown in the latitude-pressure plane in Fig. 10. This oscillation is characterized by predomi- 
(a)

MTM spectrum

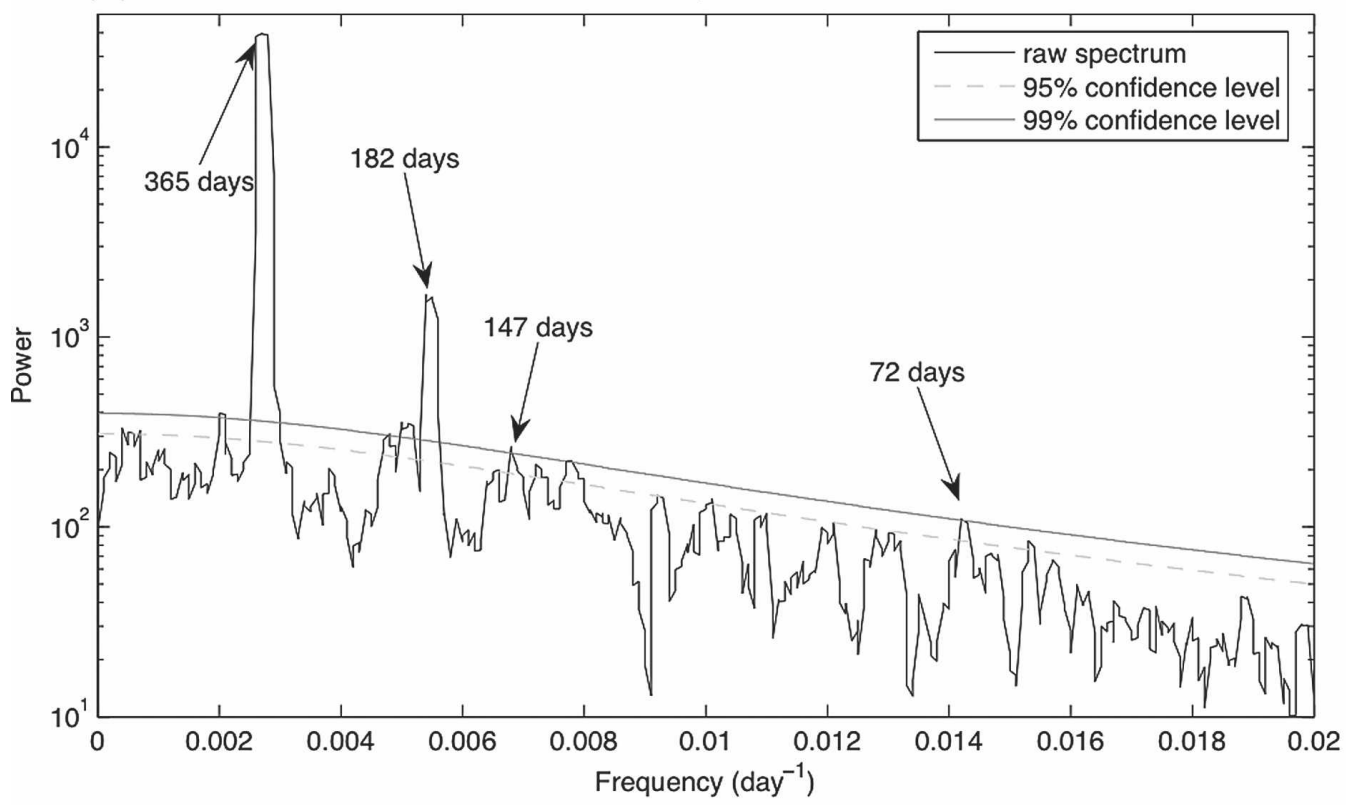

(b)

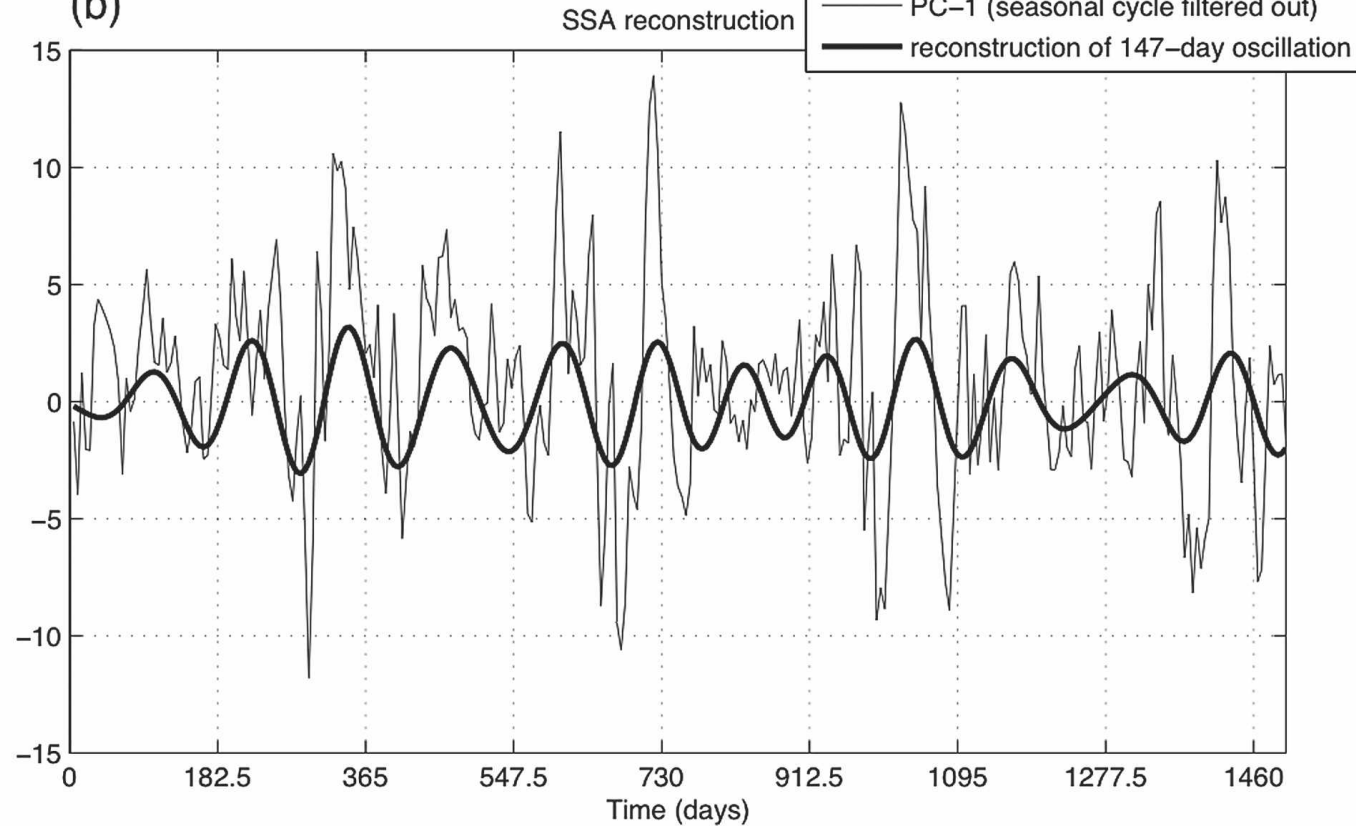

FIG. 9. Estimates of oscillatory components for the entire dataset, which includes all seasons (see text for details): (a) same as in Fig. 8b, with lines defined in the legend; (b) a 5-yr-long (1 Mar 1982-28 Feb 1987) sample time series of SSA-reconstructed components of the 147-day oscillation (heavy line) and the raw dataset's PC-1 (light line), with the seasonal cycle filtered out.

nantly equivalent barotropic anomalies that propagate northward. Evidence for poleward propagation of zonal-flow anomalies was presented by Feldstein (1998). The overall pattern of the 72-day oscillation in zonal-mean flow (not shown) is similar to that of its lower-frequency companion, except that only the low- latitude anomalies at the upper levels exhibit northward propagation, while middle- and high-latitude anomalies are stationary. This difference between the spatiotemporal patterns of the 147- and 72-day oscillations provides additional evidence that the two represent different modes of variability. 


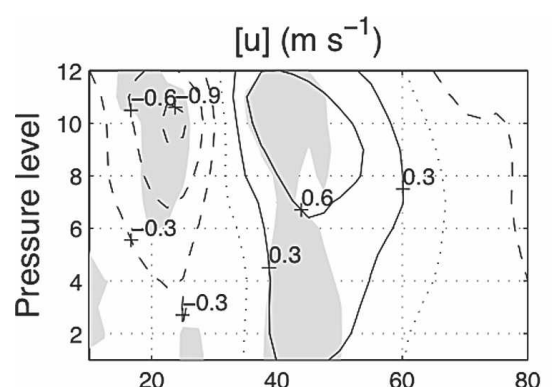

(a)

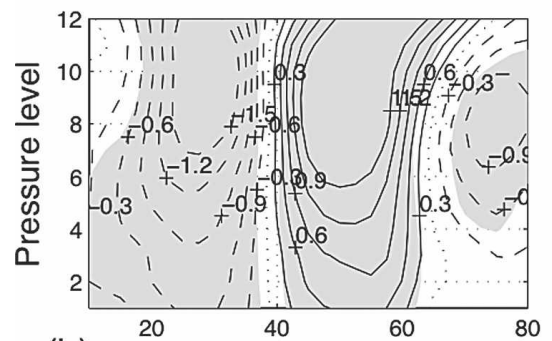

(b)

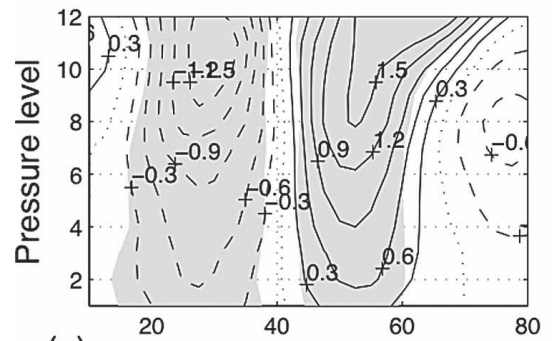

(c)

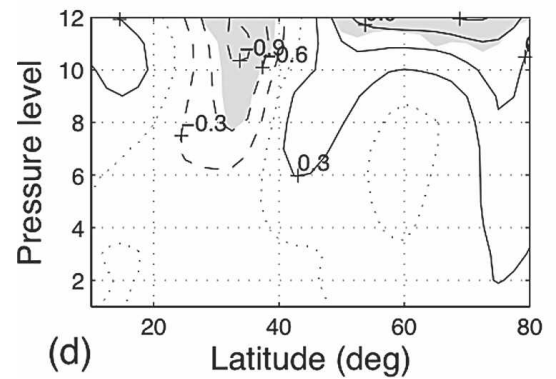

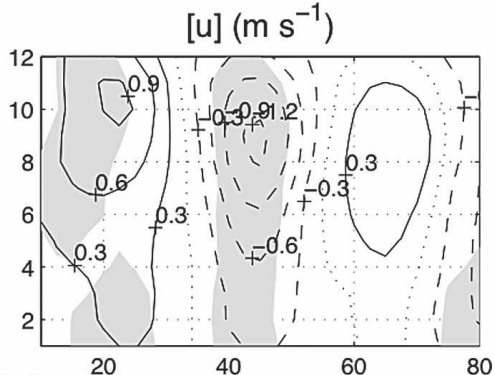

(e)

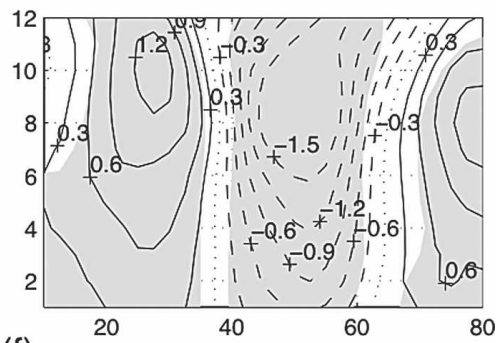

(f)

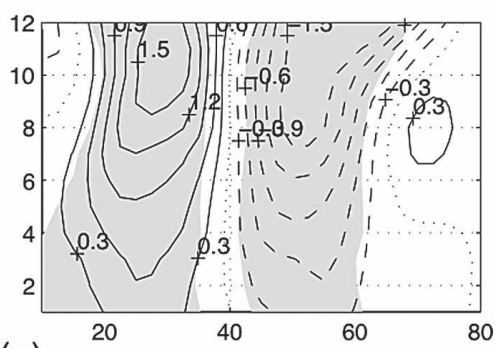

(g)

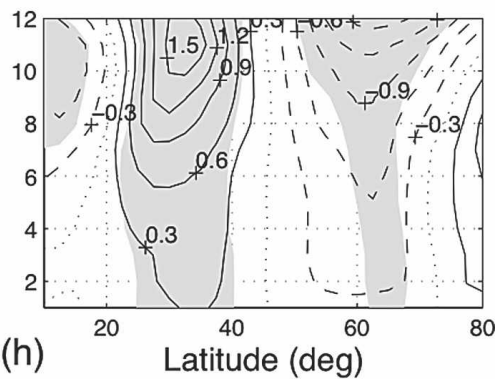

FIG. 10. Composite cycle of the 147-day oscillation in the zonal-mean zonal flow. (a)-(h) Phase categories 1-8 of the oscillation, respectively. Same units and conventions as in Fig. 5.

To examine the oscillations' horizontal pattern, we computed their composites using 700-hPa height anomalies. The patterns at the 500- and 250-hPa levels (not shown) are essentially the same.

Composites for the two oscillations have similar overall patterns in the horizontal as well. The evolution of the 72-day oscillation is displayed in Fig. 11. This oscillation is characterized by the same main centers of action over the Pacific and Atlantic Oceans as in Fig. 6 and thus resembles the AO. Its zonally averaged pattern is stationary, while poleward propagation occurs in the Atlantic sector; the latter is compensated, in the zonal averages, by anomalies elsewhere.

This poleward propagation in the Atlantic sector is most pronounced during certain phases of the oscillation. For example, the positive anomaly over the North Atlantic during phase 1 of the oscillation (Fig. 11a) moves northward and grows in size, occupying the Arctic region by the time the oscillation reaches its second phase (Fig. 11b), while a negative anomaly emerges over the North Atlantic. Similarly, a negative anomaly propagates northward, while a new positive anomaly 


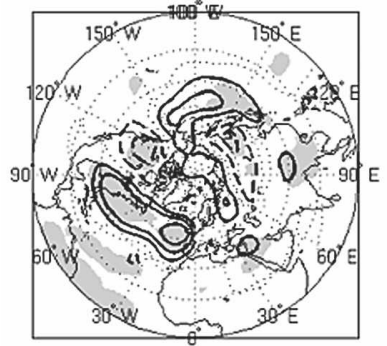

(a)

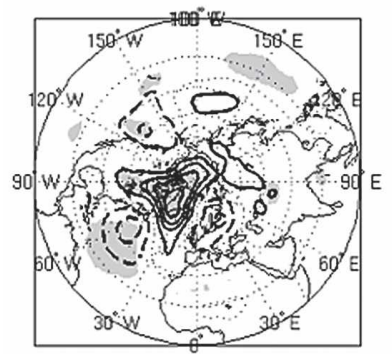

(b)

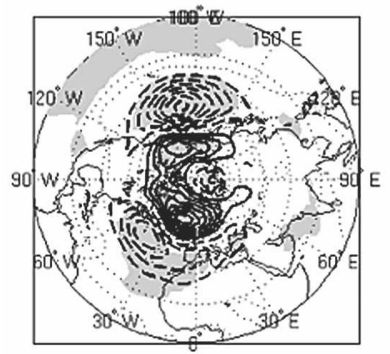

(c)

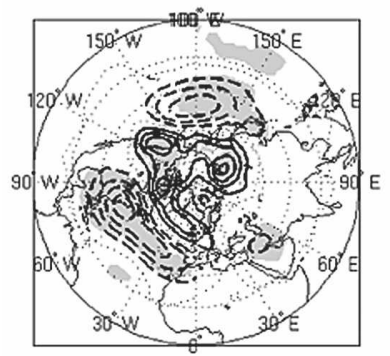

(d)

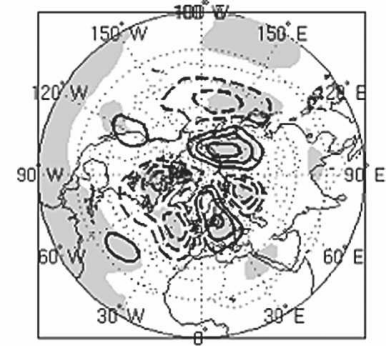

(e)

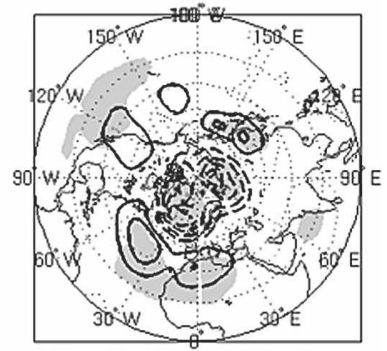

(f)

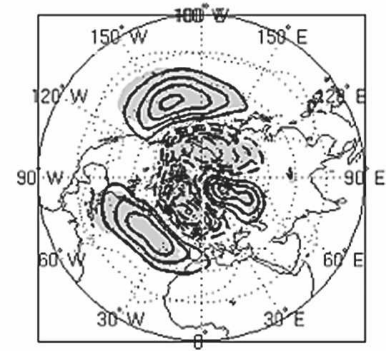

(g)

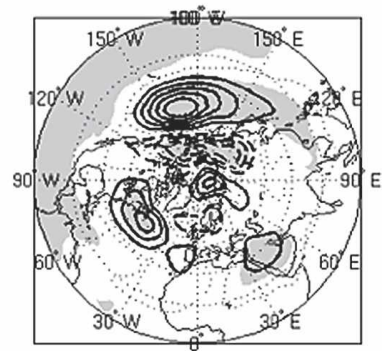

(h)

FIG. 11. Same as in Fig. 10, but for the 72-day oscillation's geopotential height anomalies at $700 \mathrm{hPa}(\mathrm{m})$. Contour interval $\mathrm{CI}=5 \mathrm{~m}$; negative contours dashed, zero contour dotted.

forms over the North Atlantic through phases 4, 5, and 6 of the oscillation (Figs. 11d-f).

The oscillation pattern over the Atlantic Ocean strongly resembles that of Plaut and Vautard's (1994) 70-day oscillation. In particular, phases 8, 1, 2, 3 (Figs. $11 \mathrm{~h}, \mathrm{a}, \mathrm{b}, \mathrm{c})$ of our 72 -day oscillation are analogous to phases $5-8$ of the oscillation found by the latter authors

(see their Fig. 7). The positive anomaly over the North Atlantic (Fig. 11h) grows in amplitude and propagates poleward through phases 1-3 (Figs. 11a-c). In contrast to Plaut and Vautard's (1994) findings, our oscillation is characterized by large-amplitude anomalies over the North Pacific Ocean as well.

\section{Multiple flow regimes and low-frequency oscillations}

The spatial patterns of the regimes in Figs. 5 and 6 are similar to the extreme phases of the low-frequency oscillations shown in Figs. 10 and 11. This similarity suggests a complementarity between the two distinct descriptions of midlatitude LFV, eposodic and oscillatory. Such a complementarity was proposed by Ghil et al. (1991) and Kimoto and Ghil (1993b), and examined in detail by Plaut and Vautard (1994) for the NH weather regimes and intraseasonal oscillations, as well as by Koo et al. (2003) for the SH zonal-flow vacillation.

The relationship between the regimes and lowfrequency oscillations detected in sections 3 and 4 is illustrated in Fig. 12. The results for the 147-day oscillation are presented in the left column (Figs. 12a-c), while those for the 72-day oscillation are in the right column (Figs. 12d-f). In Figs. 12a,b and Figs. 12d,e, we show the conditional probability of regime occurrence given the knowledge of a given, 147- or 72-day, oscillation's phase category, for regimes A and B. We find that these regimes are associated with phases $1(\mathrm{~A})$ and 6 (B) of the 147-day signal and phases 7 (A) and 4 (B) of the 72-day signal. The corresponding conditional probabilities, $\left(p_{\mathrm{A}}, p_{\mathrm{B}}\right)(147$-day $)=(0.28,0.23)$ and $\left(p_{\mathrm{A}}\right.$, $\left.p_{\mathrm{B}}\right)(72$-day $)=(0.26,0.23)$ are also higher than the unconditional probabilities $(0.2,0.16)$ for regimes (A, B), respectively.

The conditional probabilities of regime occurrence at positive lead times given the knowledge of a given oscillation's phase category at time zero (not shown) can be computed in an analogous fashion. The results are qualitatively and quantitatively similar to those of Plaut and Vautard (1994, their Fig. 17) and Koo et al. (2003, their Fig. 12); see also Ghil and Robertson (2002, their Fig. 5). The low-frequency oscillation's phase information can thus be used for long-range prediction of a given regime occurrence; see also Lott et al. (2001; $2004 a, b)$ for shorter period, intraseasonal oscillations.

To examine further the relationships between regimes and oscillations, we computed, for both oscillations, the composite phase velocity in the plane spanned by PC- 1 and its tendency. Both PC- 1 and the tendency time series were normalized by their respec- 


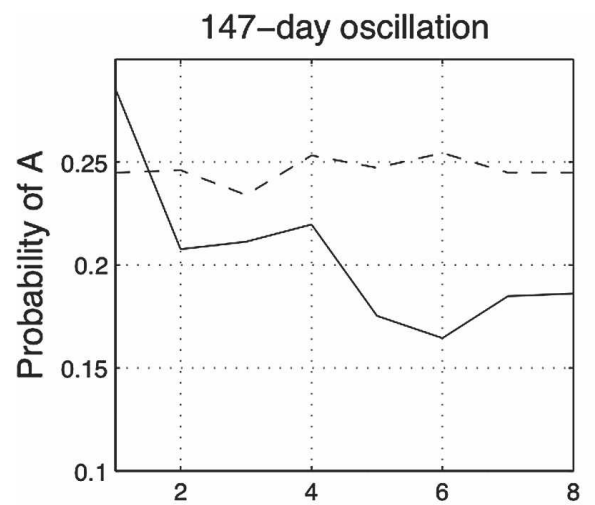

(a)

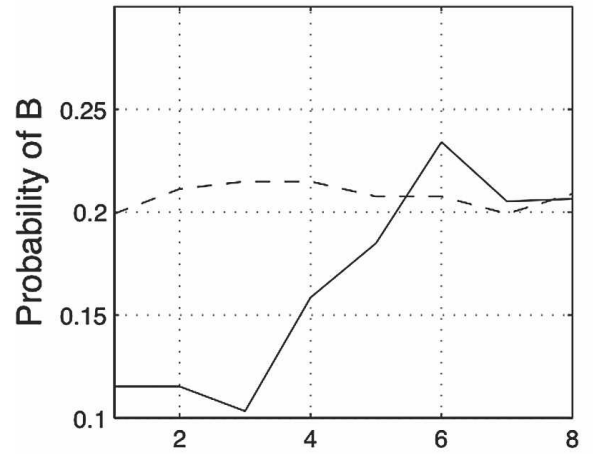

(b)

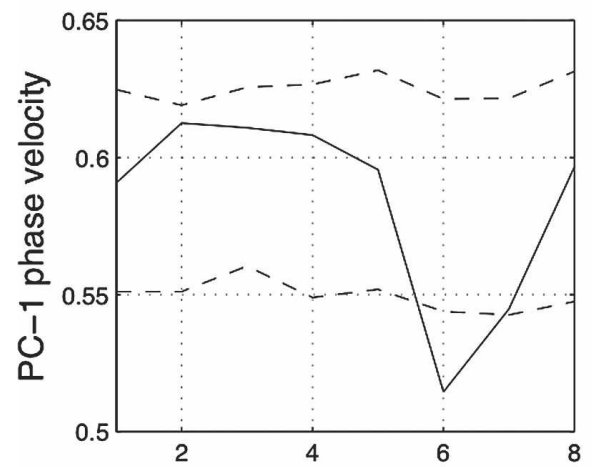

(c) Phase category of oscillation

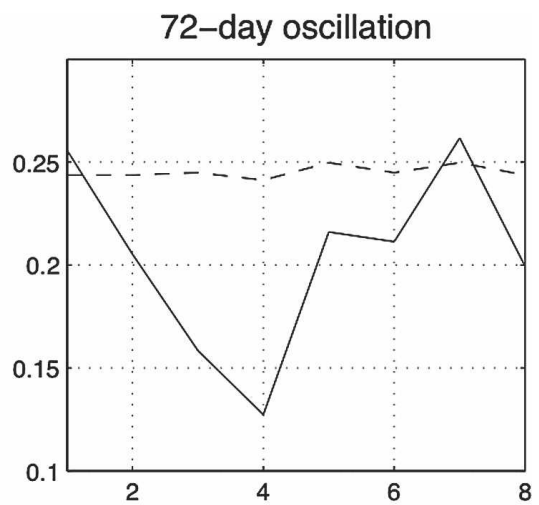

(d)

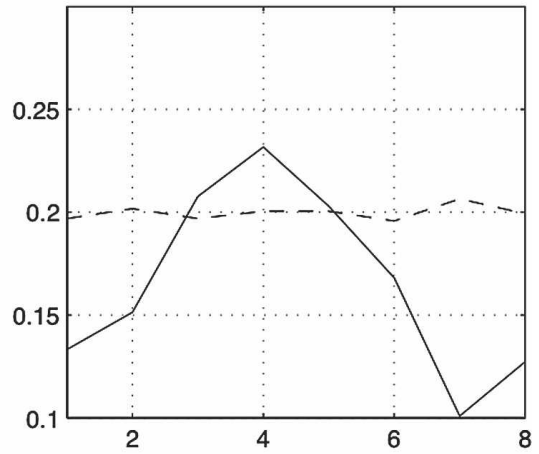

(e)

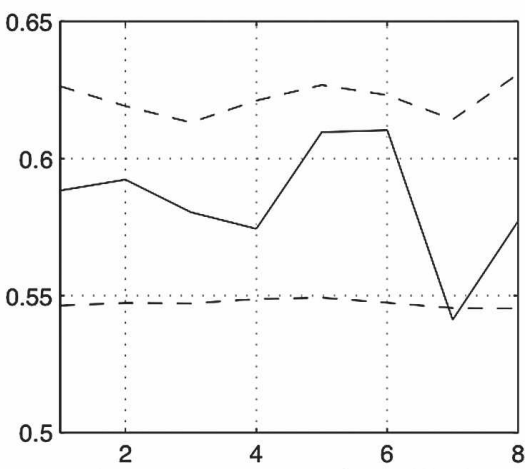

(f) Phase category of oscillation

FIG. 12. Multiple flow regimes and low-frequency oscillations: (a) probability of regime A occurrence given a phase category of the 147-day oscillation (solid line); (b) same as in (a), but for regime B. The dashed line in both panels represents the $95 \%$ confidence level. (c) Phase velocity in the plane spanned by PC-1 and its tendency, as a function of the 147-day oscillation phase category. The tendency is defined by the centered finite difference between two PC-1 values one day apart, divided by the time elapsed. Both PC-1 and the tendency time series are normalized by their respective std devs, prior to computing the phase velocity. Dashed lines represent the 5th and 95th percentile of the associated random distribution. (d)-(f) Same as in (a)-(c), but for the 72-day oscillation.

tive standard deviations; with this normalization, a purely sinusoidal oscillation has a constant phase velocity. Figures $12 \mathrm{c}, \mathrm{f}$ show the phase velocities so defined as a function of the phase category of the 147- and 72-day oscillations, respectively. We find that the phase velocity has two minima: at phases 1 and 6 of the 147-day mode, and at phases 4 and 7 of the 72-day mode; of the two, the second minimum in each pair is statistically 
significant at the $95 \%$ level. These phase-velocity minima correspond exactly to the probability maxima associated with regimes A and B in Figs. 12a,b and 12d,e. Legras and Ghil (1985) first pointed out this connection between slowing down of trajectories and regime centroids in an intermediate $\mathrm{NH}$ model with topography.

The regimes here, too, are thus related to slow phases of either oscillation. Slowing down of the phase-space trajectory is also consistent with the increased persistence associated with either regime (see Fig. 4d).

More specifically, a statistically significant slow-down of phase velocity is associated with regime A for the 72-day oscillation (cf. Figs. 12d and 12f) and with regime B for the 147-day oscillation (cf. Figs. 12b and 12c). This pairwise association between one regime and one oscillation is also consistent with the spatial patterns of regimes and oscillations. Namely, regime $\mathrm{B}$ has a more zonally symmetric character than regime A (see Fig. 6). Furthermore, the Pacific and Atlantic anomalies in regime $\mathrm{B}$ events are of the same sign and comparable magnitude, whereas the anomalies in regime A events occur predominantly in one or the other region (appendix B). Likewise, the 147-day oscillation is more zonally symmetric than the 72-day oscillation: the former is characterized by poleward propagation in both the Atlantic and Pacific sectors (see Fig. 10), while the latter oscillation's poleward propagation in the Atlantic sector is compensated elsewhere (Fig. 11).

\section{Concluding remarks}

\section{a. Summary}

We have studied Northern Hemisphere (NH) lowfrequency variability (LFV) using 55-yr-long daily NCEP-NCAR reanalysis datasets for the zonal and meridional wind and geopotential height anomalies (Kalnay et al. 1996). We have found that the NH zonalmean zonal flow is characterized by the presence of multiple flow regimes (section 3), as well as intraseasonal oscillations (section 4), and established relationships between these two complementary descriptions of LFV (Ghil et al. 1991; Kimoto and Ghil 1993b; Ghil and Robertson 2002) in section 5.

In the reduced phase space spanned by the two leading EOFs of the zonal-mean zonal flow, the probability density function (PDF) is non-Gaussian (Fig. 2), with multiple maxima present in the angular PDF (Fig. 2c) and significant ridging in the Euclidean PDF (Fig. 2a); the former emphasizes changes in the zonal-jet profile, rather than changes in jet strength. In contrast, the Euclidean PDF for the quasi-stationary (QS) data subset (Mo and Ghil 1988; Koo et al. 2003) is significantly bimodal (Fig. 4a). The persistence probability (Vautard et al. 1988) is highly bimodal as well (Figs. 4c), and the probability maxima in Fig. 4c coincide with PDF maxima in Fig. 4a. Two distinct regimes are thus identified, which are characterized by anomalously persistent flow patterns (Fig. 4d).

Composites of the two regimes show anomalies that correspond primarily to changes in the midlatitude jet position: in regime A the jet is displaced poleward, while in regime $\mathrm{B}$ it is displaced equatorward relative to its climatological position (Fig. 5). The regimes' patterns in the horizontal plane strongly resemble two opposite phases of the Arctic Oscillation (AO; Deser 2000; Thompson and Wallace 2000; Thompson et al. 2000): regime A is characterized by positive height anomalies over the midlatitude Atlantic and Pacific Oceans, and a belt of negative anomalies over the Arctic Ocean, while regime B has roughly the opposite features (Fig. 6).

We found (see appendix B) that the majority of the maps used to compute the regime B composite do possess same-sign anomalies over the Atlantic and Pacific Oceans, while only a third of the maps for regime A satisfy this property. The common spatial pattern of most regime B maps clearly resembles the AO regime found by Kondrashov et al. (2004) in the three-level quasigeostrophic model of Marshall and Molteni (1993). Kravtsov et al. (2005b) have associated this regime with an unstable equilibrium in that model's phase space. We conclude, therefore, that regime B is likely to arise from nonlinear atmospheric dynamics.

The regime anomalies are found to be maintained by synoptic eddies and damped by lower frequency transients, consistent with previous studies (Feldstein and Lee 1996, 1998; Koo 2001; Koo et al. 2003; Lorenz and Hartmann 2003). Furthermore, the eddy momentum flux due to interactions between the nonzonal part of the anomalies associated with each regime and the climatological stationary wave tends to induce a poleward drift of regime anomalies (Feldstein 1998; Lorenz and Hartmann 2003; see Fig. 7 and section 3c here).

Two advanced spectral analysis techniques, multichannel singular spectrum analysis (M-SSA) and the multitaper method (MTM) have been applied to the leading principal components (PCs) time series of the zonal-mean zonal flow. Both M-SSA and MTM spectra exhibit two common oscillatory modes, with statistically significant peaks near 147 and 72 days (Fig. 8). Both oscillations are characterized by anomalies that have a predominantly equivalent barotropic vertical structure and persist throughout the year, as shown in Fig. 9 for the 147-day oscillation. The 147-day oscillation's zonal-flow anomalies propagate poleward 
(Fig. 10), while the 72-day oscillation's pattern is stationary in middle and high latitudes, with some poleward propagation in the low-latitudes' upper levels (not shown). The horizontal patterns of the two oscillations are both reminiscent of the AO (Fig. 11). Over the North Atlantic, the 72-day oscillation's pattern closely resembles Plaut and Vautard's (1994) 70-day oscillation, with similar variations in the jet position and intensity over this ocean basin.

The two zonal-flow regimes are found to be associated with slow phases of either oscillation (Fig. 12): the conditional probabilities of a given regime occurrence when assuming the phase category of a given oscillation are significantly higher than the corresponding unconditional probabilities (see Figs. 12a,b for regime A and Figs. 12d,e for regime B). This provides hope for improved long-range forecasts of zonal-flow regimes (see also Plaut and Vautard 1994; Ghil and Robertson 2002; Koo et al. 2003).

\section{b. Discussion}

Our statistical analysis has identified connections between multiple regimes and low-frequency oscillations; these connections may be helpful for improved prediction of regime anomalies. The present results, though, do not provide a definitive explanation for the dynamical reasons behind or causal relationships between the regimes and oscillations. It is possible that our oscillations are dominated by linear dynamics and that the nonlinear effects, such as anomalous slowdown of trajectories during certain phases of either oscillation, are secondary; the regimes in this interpretation are mere manifestations of these slight nonlinear modifications of linear wave dynamics. Alternatively, the regimes may reflect separate dynamical entities, such as multiple equilibria in the midlatitude atmosphere's phase space, while linear wave trajectories are deflected and altered in the neighborhood of the regimes. Our results indicate that at least one of the zonal-flow regimes, regime $\mathrm{B}$, may be associated with such an unstable fixed point (see section 6a). Finally, the regimes and oscillations may represent a genuinely nonlinear combination associated with hetero- or homoclinic orbits (Kimoto and Ghil 1993; Itoh and Kimoto 1999; Crommelin 2003; Kondrashov et al. 2004; Selten and Branstator 2004).

Our results emphasize zonally symmetric aspects of $\mathrm{NH} \mathrm{LFV} \mathrm{and} \mathrm{are} \mathrm{qualitatively} \mathrm{consistent} \mathrm{with} \mathrm{those} \mathrm{for}$ the Southern Hemisphere (SH) LFV (Koo 2001; Koo and Ghil 2002; Koo et al. 2003), despite the presence of the $\mathrm{NH}$ topography. The role of topography in $\mathrm{NH}$ LFV has been explored in a sequence of papers (Charney and DeVore 1979; Pedlosky 1981; Legras and Ghil 1985; Ghil and Robertson 2000, and references therein). The particular theory pursued in these papers relies on a combination of topographic resonance and barotropic instability and was used to explain the presence of multiple flow regimes in NH flow (Benzi et al. 1986; Ghil and Childress 1987, chapter 6; Mo and Ghil 1988; Cheng and Wallace 1993; Kimoto and Ghil 1993a,b; Hansen and Sutera 1995; Smyth et al. 1999). The latter regimes were heretofore identified by clustering analysis on a subspace spanned by EOFs of horizontal (latitude-longitude) fields (Mo and Ghil 1988; Molteni et al. 1990) or by using a highly nonlinear separating functional, the wave amplitude index (Benzi et al. 1986), on this subspace. The regimes so determined had a large degree of zonal asymmetry, with features mostly concentrated in two sectors, Atlantic and Pacific, and were often associated with extreme phases of intraseasonal oscillations that had periods of 15-40 days (Ghil and Robertson 2002; Lott et al. 2004a,b).

In contrast, the regimes identified in the present paper are much more zonally symmetric. This fundamental difference may be due to our concentrating on longer time scales, of several months, and choosing a different approach to the preliminary data compression. To wit, we set out to emphasize the zonal-mean flow by using its monthly mean EOFs (see section 2b), while earlier studies employed the EOFs of the horizontal fields, typically low-pass filtered at 10 days. The two procedures are complementary, with the present method tracking the lowest frequency modes of subannual variability, which have a tendency to be more zonally symmetric (Kutzbach 1970; Mo and Ghil 1988; Thompson and Wallace 2000), while the previous methodology concentrated on sectorial regimes that have somewhat shorter time scales and, presumably, different dynamics associated with them.

The observational findings of Koo and coauthors (Koo 2001; Koo et al. 2003) and ours are consistent with recent theories for the origin and maintenance of annular modes. Koo and Ghil (2002) presented such a theory using a nonlinear, low-order model. While Cehelsky and Tung (1987) argued that multiple regimes in low-order models may be artifacts of extreme truncation, Kravtsov et al. (2003, 2005a) have essentially recovered Koo and Ghil's (2002) bimodality results in a higher dimensional setting. In Kravtsov et al. (2003, 2005a), the behavior of a two-layer atmospheric model with fairly high spatial resolution was studied as a function of the surface friction, expressed in terms of the barotropic spindown time scale $t_{\mathrm{f}}$. For high values of surface friction, $2<t_{\mathrm{f}}<6$ days, this model is characterized by unimodal behavior. As the surface friction decreases to more realistic values, two regimes are 
found, which differ by the meridional position of the midlatitude jet.

The model's variability in the parameter range of bimodality is characterized by irregular transitions between its high-latitude and low-latitude states, as well as by the presence of equivalent barotropic, ultra-lowfrequency oscillations with periods longer than 100 days. The two states become increasingly more separated in latitude as $t_{\mathrm{f}}$ increases further, while the periods of the ultra-low-frequency modes decrease.

Lorenz and Hartmann $(2001,2003)$ estimate the surface friction parameter to be $t_{\mathrm{f}} \approx 9$ days for the SH and $t_{\mathrm{f}} \approx 7$ days for the $\mathrm{NH}$. These parameter values place both hemispheres in the bimodal regime, according to Kravtsov et al.'s (2005a) model results, which is consistent with the observational findings of Koo et al. (2003) and of the present paper. Furthermore, the ultra-lowfrequency oscillations in the $\mathrm{SH}$ do have slightly shorter periods, of 135 and 70 days (Koo et al. 2003), while the jet positions of the two SH regimes are located further apart in latitude than their NH counterparts. There is thus strong evidence, both observational and theoretical, that the zonal-flow behavior in both hemispheres differs substantially from the one predicted by a linear, purely noise-driven model, as proposed earlier (Kidson and Watterson 1999; Feldstein 2000).

Acknowledgments. We are grateful to three anonymous reviewers for their thoughtful comments; one of them, in particular, emphasized the connection between regime $\mathrm{A}$ and the 72-day oscillation, and between regime $\mathrm{B}$ and the 147-day oscillation. This research was supported by NSF Grants ATM-0082131 and OCE-02-221066 (MG and SK) and DOE Grant DE-FG-03-01ER63260 (SK and AWR).

\section{APPENDIX A}

\section{Linear Stochastic Model for Null Hypothesis}

To test the statistical significance of the observed PDF, we formulated a null hypothesis stating that the observed time series in the ten-dimensional space of leading zonal-flow EOFs (see sections $2 b$, c) cannot be distinguished from a linear stochastic process. The model that describes the latter was obtained following Kravtsov et al. (2005b). This model is a multilevel generalization of the one used by Koo et al. (2003). If $\mathbf{x}$ is a given vector time series, the one-level model has the general form

$$
\mathrm{d} \mathbf{x}=\mathbf{B}^{(0)} \mathbf{x} \mathrm{d} t+\mathrm{d} \mathbf{r}^{(0)},
$$

where the matrix $\mathbf{B}^{(0)}$ and the covariance matrix $\mathbf{Q} \equiv$ $\left\langle\mathbf{r}^{(0)} \mathbf{r}^{(0)^{\mathrm{T}}}\right\rangle$ of the residual forcing $\mathbf{r}^{(0)}$ are determined by least squares. If the inverse model contains a large number of variables, the statistical distribution of $\mathbf{r}^{(0)}$ at a given instant is nearly Gaussian, according to the central limit theorem (Von Mises 1964).

However, the stochastic forcing $\mathbf{r}^{(0)}$ in Eq. (A1) involves serial correlations and might also depend on the modeled process $\mathbf{x}$. We include, therefore, an additional model level to express the time increments $d \mathbf{r}^{(0)}$ as a linear function of an extended state vector $\left[\mathbf{x}, \mathbf{r}^{(0)}\right]$, and estimate this level's residual forcing $\mathbf{r}^{(1)}$. More levels are being added in the same way, until the $N$ th level's residual $\mathbf{r}^{(N+1)}$ becomes white in time, and its lag- 0 correlation matrix no longer changes if we add one more level. The complete stochastic model is thus given by

$$
\begin{aligned}
\mathrm{d} \mathbf{x} & =\mathbf{B}^{(0)} \mathbf{x} \mathrm{d} t+\mathbf{r}^{(0)} \mathrm{d} t \\
\mathrm{~d} \mathbf{r}^{(0)} & =\mathbf{B}^{(1)}\left[\mathbf{x}, \mathbf{r}^{(0)}\right] \mathrm{d} t+\mathbf{r}^{(1)} \mathrm{d} t, \\
\mathrm{~d} \mathbf{r}^{(1)} & =\mathbf{B}^{(2)}\left[\mathbf{x}, \mathbf{r}^{(0)}, \mathbf{r}^{(1)}\right] \mathrm{d} t+\mathbf{r}^{(2)} \mathrm{d} t, \\
& \cdots \\
\mathrm{d} \mathbf{r}^{(N)} & =\mathbf{B}^{(N)}\left[\mathbf{x}, \mathbf{r}^{(0)}, \mathbf{r}^{(1)}, \ldots, \mathbf{r}^{(N)}\right] \mathrm{dt}+\mathrm{d} \mathbf{r}^{(N+1)} .
\end{aligned}
$$

For the data used in this study, the optimal number of layers is equal to 3 , including the zeroth layer (that is $N=2$ ).

One hundred synthetic time series with the same length as that of the data were then generated by integrating the model (A2) with $\mathrm{d} t=1$ day, while substituting $\mathrm{d} \mathbf{r}^{(3)}$ by white-noise increments that have the estimated lag- 0 correlation matrix.

\section{APPENDIX B}

\section{On Zonal Symmetry of Regime Composites}

The regime composites A and B (Fig. 6) exhibit anomalies of the same sign over the Atlantic and Pacific sectors. Are individual regime maps characterized by the same property? To address this question, we have computed, following Deser (2000), the leading EOFs of monthly wintertime $700-\mathrm{mb}$ geopotential height anomalies for the whole $\mathrm{NH}$, as well as for its Atlantic $\left(90^{\circ} \mathrm{W}-90^{\circ} \mathrm{E}\right)$ and Pacific $\left(90^{\circ} \mathrm{E}-270^{\circ} \mathrm{W}\right)$ sectors.

Regression maps of monthly height anomalies onto the hemispheric and sectorial leading PCs are plotted in Fig. B1. Figure B1a, based on hemispheric results, shows an annular mode with a negative center over the Arctic and positive anomalies in both the Atlantic and Pacific sectors; the maps based on Atlantic-only (Fig. B1b) and Pacific-only (Fig. B1c) PCs show anomalies 


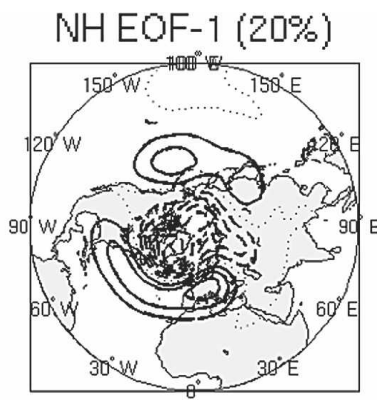

(a)

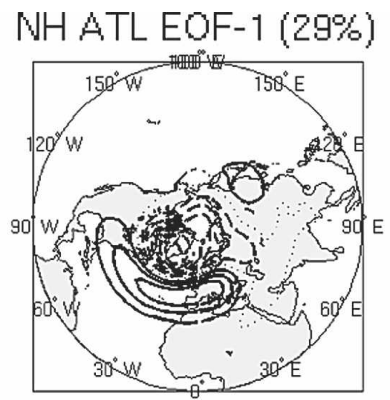

(b)

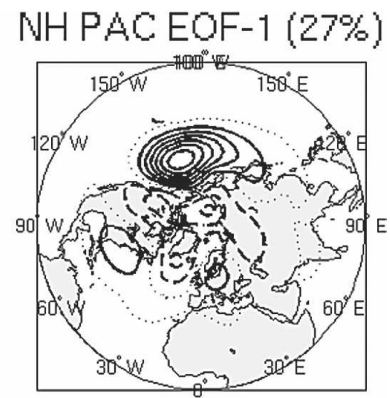

(c)

FIG. B1. Regression maps of wintertime 700-mb height anomalies onto the leading PCs computed for (a) the whole NH; (b) the North Atlantic sector; and (c) the North Pacific sector; CI = $10 \mathrm{~m}$, negative contours dashed, zero contour dotted.

that are mostly confined within their respective base sectors. We have formed daily time series of hemispheric and sectorial patterns by projecting daily data onto the corresponding monthly mean EOFs. The cor- relations between these time series are $C($ Hemisphere, Atlantic $)=0.92, C($ Hemisphere, Pacific $)=0.47$, and $C($ Atlantic, Pacific $)=0.15$. A small correlation between the Atlantic and Pacific time series shows a weak
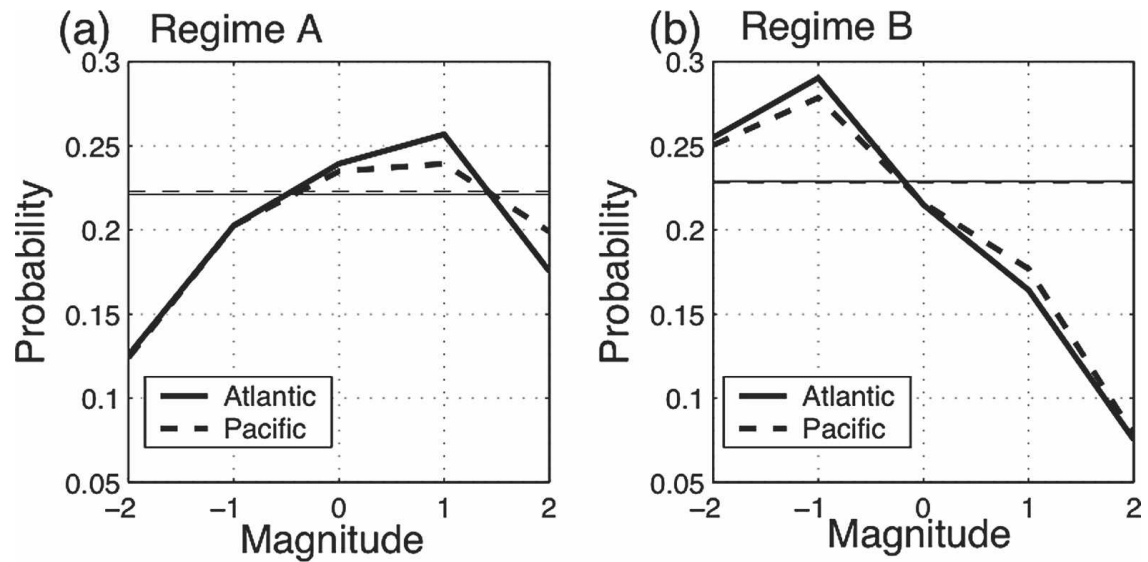

\section{(c) Probability}
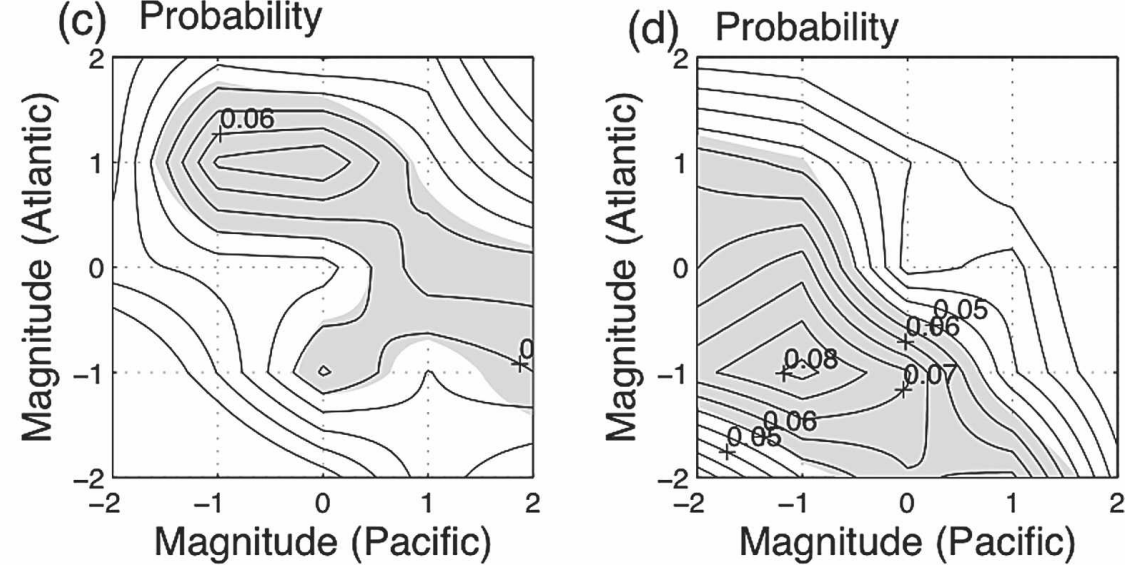

FIG. B2. Conditional probabilities of regime occurrence given magnitude categories of the leading Atlantic and Pacific PCs of Fig. 13; (a), (c) for regime A; (b), (d): for regime B. Light horizontal lines in (a), (b) and shading in (c), (d) show the $95 \%$ confidence level based on a linear bivariate stochastic process null hypothesis. 
teleconnectivity, at best, between the NH Atlantic and Pacific regions (Deser 2000).

We now divide each of the Atlantic and Pacific time series into five equally populated categories $-2,-1,0$, 1,2 , sorted by the magnitude of the corresponding anomaly, and compute the conditional probability of regimes $\mathrm{A}$ and $\mathrm{B}$ to occur within a given range of anomaly magnitude (Fig. B2). Figures B2a,c summarize the results for regime A, and Figs. B2b,d for regime B. All panels also show $95 \%$ confidence levels based on Monte Carlo tests that use 100 bivariate surrogate representations, based on a three-level linear regression fit (see appendix A) of the Atlantic and Pacific time series.

Figures B2a,b show that regime A occurs preferentially during categories 0 and 1 , while regime $\mathrm{B}$ occupies mostly categories -2 and -1 of both Atlantic and Pacific time series. In Figs. B2c,d, we plot conditional probabilities given simultaneous Atlantic and Pacific categories. For regime B (Fig. B2d), a significant fraction of regime events is characterized by anomalies of the same sign and comparable magnitude in both the Atlantic and Pacific sectors. This regime composite (Fig. 6b) represents, therefore, a recurrent circulation pattern that does extend over the entire $\mathrm{NH}$.

The situation is much different, however, for regime A (Fig. B2c). This regime's probability of occurrence is larger during (Pacific, Atlantic) categories $(-1,1),(0$, $-1),(0,1),(1,0)$, and $(2,0)$. Computing the same conditional probability (not shown) based on the QS subset of the full dataset (section $3 b$ ) demonstrates that only the enhanced probabilities of categories $(0,1),(1,0)$, and $(2,0)$ are due to QS anomalies. The full composite of regime A (Fig. 6a) is thus primarily the sum of two subsets of recurrent circulation maps, which are characterized by a large anomaly in one of the basins, and a smaller anomaly in the other.

\section{REFERENCES}

Allen, M. R., and A. W. Robertson, 1996: Distinguishing modulated oscillations from colored noise in multivariate data sets. Climate Dyn., 12, 775-784.

Benzi, R., P. Malguzzi, A. Speranza, and A. Sutera, 1986: The statistical properties of general atmospheric circulation: Observational evidence and a minimal theory of bimodality. Quart. J. Roy. Meteor. Soc., 112, 661-674.

Broomhead, D. S., and G. P. King, 1986: Extracting qualitative dynamics from experimental data. Physica D, 20, 217-236.

Cehelsky, P., and K. K. Tung, 1987: Theories of multiple equilibria and weather regimes-A critical reexamination. Part II: Baroclinic two-layer models. J. Atmos. Sci., 44, 3282-3303.

Charney, J. G., and J. G. DeVore, 1979: Multiple flow equilibria in the atmosphere and blocking. J. Atmos. Sci., 36, 762-779.

Cheng, X. H., and J. M. Wallace, 1993: Analysis of the northernhemisphere wintertime $500-\mathrm{hPa}$ height field spatial patterns. J. Atmos. Sci., 50, 2674-2696.
Crommelin, D. T., 2003: Regime transitions and heteroclinic connections in a barotropic atmosphere. J. Atmos. Sci., 60, 229-246.

Deser, C., 2000: On the teleconnectivity of the "Arctic Oscillation." Geophys. Res. Lett., 27, 779-782.

Dettinger, M. D., and M. Ghil, 1998: Seasonal and interannual variations of atmospheric $\mathrm{CO}_{2}$ and climate. Tellus, 50B, 1-24.

Dole, R. M., and N. D. Gordon, 1983: Persistent anomalies of the extratropical Northern Hemisphere wintertime circulation - Geographical distribution and regional persistence characteristics. Mon. Wea. Rev., 111, 1567-1586.

Feldstein, S. B., 1998: An observational study of intraseasonal poleward propagation of zonal mean flow anomalies. J. Atmos. Sci., 55, 2516-2529.

- 2000: Is interannual zonal mean flow variability simply climate noise? J. Climate, 13, 2356-2362.

_ an aquaplanet GCM. J. Atmos. Sci., 53, 3541-3555.

— , and - 1998: Is the atmospheric zonal index driven by an eddy feedback? J. Atmos. Sci., 55, 3077-3086.

Fraedrich, K., 1986: Estimating the dimension of weather and climate attractors. J. Atmos. Sci., 43, 419-432.

Ghil, M., and S. Childress, 1987: Topics in Geophysical Fluid Dynamics: Atmospheric Dynamics, Dynamo Theory and Climate Dynamics. Springer-Verlag, 485 pp.

— , and K. C. Mo, 1991a: Intraseasonal oscillations in the global atmosphere. Part I: Northern Hemisphere and Tropics. $J$. Atmos. Sci., 48, 752-779.

$\longrightarrow$, and _ 1991b: Intraseasonal oscillations in the global atmosphere. Part II: Southern Hemisphere. J. Atmos. Sci., 48, 780-790.

_ , and A. W. Robertson, 2000: Solving problems with GCMs: General circulation models and their role in the climate modeling hierarchy. General Circulation Model Development: Past, Present and Future, D. Randall, Ed., Academic Press, 285-325.

—_, and _ 2002: "Waves" vs. "particles" in the atmosphere's phase space: A pathway to long-range forecasting? Proc. Natl. Acad. Sci., 99 (Suppl. 1), 2493-2500.

_ M. Kimoto, and J. D. Neelin, 1991: Nonlinear dynamics and predictability in the atmospheric sciences. Rev. Geophys., 29, $46-55$.

—, and Coauthors, 2002: Advanced spectral methods for climatic time series. Rev. Geophys., 40, 1-41.

Hansen, A. R., and A. Sutera, 1995: The probability density distribution of planetary-scale atmospheric wave amplitude revisited. J. Atmos. Sci., 52, 2463-2472.

Hartmann, D. L., 1995: A PV view of zonal flow vacillation. $J$. Atmos. Sci., 52, 2561-2576.

—, and F. Lo, 1998: Wave-driven zonal flow vacillation in the Southern Hemisphere. J. Atmos. Sci., 55, 1303-1315.

Hsu, C. J., and F. Zwiers, 2001: Climate change in recurrent regimes and modes of Northern Hemisphere atmospheric variability. J. Geophys. Res., 106, 20 145-20 159.

Itoh, H., and M. Kimoto, 1999: Weather regimes, low-frequency oscillations, and principal patterns of variability: A perspective of extratropical low-frequency variability. J. Atmos. Sci., 56, 2684-2705.

Kalnay, E., and Coauthors, 1996: The NCEP/NCAR 40-Year Reanalysis Project. Bull. Amer. Meteor. Soc., 77, 437-471.

Kalnay-Rivas, E., and L. O. Merkine, 1981: A simple mechanism for blocking. J. Atmos. Sci., 38, 2077-2091.

Keppenne, C. L., and M. Ghil, 1993: Adaptive filtering and prediction of noisy multivariate signals: An application to sub- 
annual variability in atmospheric angular momentum. Int. J. Bifurcation Chaos, 3, 625-634.

Kidson, J. W., and I. G. Watterson, 1999: The structure and predictability of the "high-latitude mode" in the CSIRO9 general circulation model. J. Atmos. Sci., 56, 3859-3873.

Kimoto, M., and M. Ghil, 1993a: Multiple flow regimes in the Northern Hemisphere winter. Part I: Methodology and hemispheric regimes. J. Atmos. Sci., 50, 2625-2643.

— Hemisphere winter. Part II: Sectorial regimes and preferred transitions. J. Atmos. Sci., 50, 2645-2673.

Kondrashov, D., K. Ide, and M. Ghil, 2004: Weather regimes and preferred transition paths in a three-level quasi-geostrophic model. J. Atmos. Sci., 61, 568-587.

Koo, S., 2001: Nonlinear aspects of atmospheric zonal-flow vacillation. Ph.D. thesis, University of California, Los Angeles, 163 pp. , and M. Ghil, 2002: Successive bifurcations in a simple model of atmospheric zonal-flow vacillation. Chaos, 12, 300-309.

— A. A. Robertson, and M. Ghil, 2003: Multiple regimes and low-frequency oscillations in the Southern Hemisphere's zonal-mean flow. J. Geophys. Res., 107, 4596, doi:10.1029/ 2001JD001353.

Kravtsov, S., A. W. Robertson, and M. Ghil, 2003: Low-frequency variability in a baroclinic $\beta$-channel with land-sea contrast. $J$. Atmos. Sci., 60, 2267-2293.

,$- \ldots$, and,- 2005 a: Bimodal behavior in the zonal-mean flow of a baroclinic $\beta$-channel model. J. Atmos. Sci., 62, 1746-1769.

— , D. Kondrashov, and M. Ghil, 2005b: Multilevel regression modeling of nonlinear processes: Derivation and applications to climatic variability. J. Climate, 18, 4404-4424.

Kutzbach, J. E., 1970: Large-scale features of monthly mean Northern Hemisphere anomaly maps of sea-level pressure. Mon. Wea. Rev., 98, 708-716.

Legras, B., and M. Ghil, 1985: Persistent anomalies, blocking and variations in atmospheric predictability. J. Atmos. Sci., 42, 433-471.

Lorenz, D. J., and D. L. Hartmann, 2001: Eddy-zonal flow feed back in the Southern Hemisphere. J. Atmos. Sci., 58, 3312-3327.

— and — 2003: Eddy-zonal flow feedback in the Northern Hemisphere winter. J. Climate, 16, 1212-1227.

Lott, F., A. W. Robertson, and M. Ghil, 2001: Mountain torques and atmospheric oscillations. Geophys. Res. Lett., 28, 1207-1210.

$\ldots, \ldots$, and $—, 2004 \mathrm{a}$ : Mountain torques and Northern Hemisphere low-frequency variability. Part I: Hemispheric aspects. J. Atmos. Sci., 61, 1259-1271.

,$- \ldots$, and $\longrightarrow, 2004 \mathrm{~b}$ : Mountain torques and Northern Hemisphere low-frequency variability. Part II: Regional aspects. J. Atmos. Sci., 61, 1272-1283.

Mann, M. E., and J. M. Lees, 1996: Robust estimation of background noise and signal detection in climatic time series. Climate Change, 33, 409-445.

Marshall, J., and F. Molteni, 1993: Toward a dynamical understanding of atmospheric weather regimes. J. Atmos. Sci., 50, 1792-1818.

Mo, K., and M. Ghil, 1988: Cluster analysis of multiple planetary flow regimes. J. Geophys. Res., 93D, $10927-10952$.

Molteni, F., S. Tibaldi, and T. N. Palmer, 1990: Regimes in the wintertime circulation over northern extratropics. 1. Observational evidence. Quart. J. Roy. Meteor. Soc., 116A, 31-67.

Moron, V., R. Vautard, and M. Ghil, 1998: Trends, interdecadal and interannual oscillations in global sea-surface temperatures. Climate Dyn., 14, 545-569.

North, G. R., T. L. Bell, R. F. Cahalan, and F. J. Moeng, 1982:
Sampling errors in the estimation of empirical orthogonal functions. Mon. Wea. Rev., 110, 699-706.

Otnes, R., and L. Enochson, 1978: Applied Time Series Analysis. Vol. I, Wiley and Sons, 449 pp.

Pedlosky, J., 1981: Resonant topographic waves in barotropic and baroclinic flows. J. Atmos. Sci., 38, 2626-2641.

Plaut, G., and R. Vautard, 1994: Spells of low-frequency variability and weather regimes in the Northern Hemisphere. J. Atmos. Sci., 51, 210-236.

Reinhold, B. B., and R. T. Pierrehumbert, 1982: Dynamics of weather regimes: Quasi-stationary waves and blocking. Mon. Wea. Rev., 110, 1105-1145.

Robertson, A. W., 2001: Influence of ocean-atmosphere interaction on the Arctic Oscillation in two general circulation models. J. Climate, 14, 3240-3254.

Robinson, W., 1991: The dynamics of low-frequency variability in a simple model of the global atmosphere. J. Atmos. Sci., 48, 429-441.

_ 1996 : Does eddy feedback sustain variability in the zonal index? J. Atmos. Sci., 53, 3556-3569.

- 2000: A baroclinic mechanism for the eddy feedback on the zonal index. J. Atmos. Sci., 57, 415-422.

Selten, F. M., and G. Branstator, 2004: Preferred regime transition routes and evidence for an unstable periodic orbit in a baroclinic model. J. Atmos. Sci., 61, 2267-2282.

Silverman, B. W., 1986: Density Estimation for Statistics and Data Analysis. Chapman and Hall, 175 pp.

Smyth, P., K. Ide, and M. Ghil, 1999: Multiple regimes in Northern Hemisphere height fields via mixture model clustering. $J$. Atmos. Sci., 56, 3704-3723.

Thompson, D. W. J., and J. M. Wallace, 2000: Annular modes in the extratropical circulation. Part I: Month-to-month variability. J. Climate, 13, 1000-1016.

,-- , and G. C. Hegerl, 2000: Annular modes in the extratropical circulation. Part II: Trends. J. Climate, 13, 1018-1036.

Thomson, D. J., 1982: Spectrum estimation and harmonic analysis. IEEE Proc., 70, 1055-1096.

_ 1990: Quadratic-inverse spectrum estimates: Application to paleoclimatology. Philos. Trans. Roy. Soc. London, A332, 539-597.

Vautard, R., and M. Ghil, 1989: Singular spectrum analysis in nonlinear dynamics, with applications to paleoclimatic time series. Physica D, 35, 395-424.

— B. Legras, and M. Deque, 1988: On the source of midlatitude low-frequency variability. Part I: A statistical approach to persistence. J. Atmos. Sci., 45, 2811-2843.

— transition matrices of atmospheric Markov chains. J. Atmos. Sci., 47, 1926-1931.

_ P. Yiou, and M. Ghil, 1992: Singular-spectrum analysis: A toolkit for short, noisy chaotic signals. Physica D, 58, 95-126.

Von Mises, R., 1964: Mathematical Theory of Probability and Statistics. Academic Press, 694 pp.

Wallace, J. M., 1983: The climatological mean stationary waves: Observational evidence. Large-Scale Dynamical Processes in the Atmosphere, B. J. Hoskins and R. P. Pearce, Eds., Academic Press, 27-53.

_, 2000: North Atlantic Oscillation/annular mode: Two paradigms-One phenomenon. Quart. J. Roy. Meteor. Soc., 126, 791-805.

Yu, J.-Y., and D. L. Hartmann, 1993: Zonal flow vacillation and eddy forcing in a simple GCM of the atmosphere. J. Atmos. Sci., 50, 3244-3259. 